Inra Prod. Anim., 2009, 22 (4), 303-316

\title{
Pratiques d'élevages et performances des génisses laitières : état des connaissances et perspectives
}

\author{
Y. LE COZLER 1, 2, J.-R. PECCATTE ${ }^{3}$, J.-Y. PORHIEL ${ }^{4}$, P. BRUNSCHWIG ${ }^{5}$, C. DISENHAUS 1,2 \\ ${ }^{1}$ INRA, UMR1080 Production du Lait, F-35590 Saint-Gilles, France \\ 2 Agrocampus Ouest, UMR1080 Production du Lait, F-35042 Rennes, France \\ 3 INRA, UE326, Le Pin au Haras, Borculo, F-61310 Exmes, France \\ ${ }^{4}$ Chambre Régionale d'Agriculture de Bretagne, Pôle Herbivores, F-29000 Quimper, France \\ 5 Institut de l'Elevage, UP CATL, 9 rue André Brouard, F-49105 Angers, France \\ Courriel : yannick.lecozler@agrocampus-ouest.fr
}

Les génisses représentent près de $50 \%$ des animaux présents dans la plupart des exploitations laitières. Les travaux publiés sur l'alimentation et l'élevage des génisses intégrant leurs effets sur la carrière de la future vache laitière sont déjà anciens. Ils méritaient d'être actualisés.

Avec l'agrandissement de la taille des troupeaux laitiers, les effectifs de génisses de renouvellement ont tendance à fortement augmenter. L'organisation du travail est souvent affectée et des simplifications de conduite alimentaire peuvent aller à l'encontre de la maîtrise du coût alimentaire et de la longévité des vaches dans l'élevage. Le troupeau de génisses est par ailleurs un bon «valorisateur» de prairies non accessibles aux vaches laitières (et/ou non retournables) et de fourrages conservés de moindre valeur alimentaire que celle nécessaire aux vaches.

Mais les conditions d'élevage de la génisse laitière ont des impacts non négligeables, sur l'animal bien sûr (production de lait, carrière...), sur l'éleveur (travail occasionné, gestion de la reproduction...) mais aussi sur l'exploitation toute entière (coûts d'élevage, rejets azotés...). De nombreuses études, tant en France qu'à l'étranger, ont souvent mis en avant leur importance économique (Heinrichs 1996, Pirlo et al 1997, Mourits et al 1999, Brisson 2006, Hadley et al 2006). La faisabilité et l'intérêt économique de la réduction de l'âge au vêlage de 36 à 24 mois chez la race Holstein est connue mais au cours des deux dernières décennies, les données des élevages suivis au contrôle laitier montrent qu'au niveau national, l'âge au $1^{\text {er }}$ vêlage n'a pratiquement pas évolué (29-30 mois en moyenne), tout comme l'importance du renouvellement. D'autres aspects liés à cet élevage sont aussi à prendre en considération. Par exemple, sur la base des références Corpen (1999), on peut estimer qu'une baisse de $10 \%$ du taux de renouvellement se traduit par une réduction variant de 265 à $335 \mathrm{~kg}$ d'azote organique produit, pour un élevage de 40 vaches laitières en production sur l'année. Soit deux hectares de surface d'épandage en moins et une économie de 9 à $200 €$ par 1000 litres produits. Cet aspect est loin d'être anodin dans des zones à forte densité animale. Enfin, le temps de travail total pour élever une génisse pour un vêlage à 24 mois depuis sa naissance est de $18 \mathrm{~h}$ en moyenne (Porhiel 2002).

L'impact des pratiques d'élevage pendant la croissance, et notamment de l'alimentation, a largement été étudié, en particulier sur l'apparition de la puberté, la mise à la reproduction et les performances ultérieures (Le Cozler et al 2008). Sur ce dernier point, la majo- rité des études s'est focalisée sur les conséquences à court terme, à savoir les performances de production et de reproduction en 1 ère lactation. On peut toutefois noter qu'il n'existe pas toujours de consensus parmi les auteurs sur l'impact des conditions d'élevage et notamment sur les possibles effets d'une alimentation trop riche sur le développement mammaire. Les effets sur le long terme sont moins connus, par les scientifiques en raison des coûts expérimentaux et les difficultés à mettre en place de telles expériences, et par les éleveurs souvent en raison du décalage dans le temps entre les pratiques sur génisses et l'impact en tant que vache. Ceci est d'autant plus vrai que la conduite des animaux adultes peut complètement occulter ce qui se passe avant le $1^{\text {er }}$ vêlage.

Cet article s'inspire d'une synthèse bibliographique réalisée sur l'effet des conditions d'élevage et l'optimisation du $1^{\text {er }}$ vêlage (Le Cozler et al 2008). Il en reprend les points principaux, complétés de données expérimentales récentes, d'observations en élevages et d'aspects non traités dans cette revue, comme par exemple l'importance du colostrum. Les objectifs principaux de 
ce travail effectué à partir de ces différentes sources d'information sont de faire ressortir les principales applications possibles en élevage, en se focalisant essentiellement sur les grandes étapes de l'élevage des génisses, mais aussi sur les facteurs clés de la réussite de la mise à la reproduction et de la maîtrise des coûts.

\section{1 / Influence des conditions de vie fotale}

Chez la génisse comme chez d'autres jeunes femelles de mammifères, les conditions de vie intra-utérine ont semble-t-il un effet possible sur les performances ultérieures, et sont notamment influencées par l'alimentation de la mère et sa parité, même si cet effet n'est pas toujours clair et évident (Gardner et al 2008).

Chez les races allaitantes, l'alimentation de la mère en fin de gestation a un effet positif sur le taux de fertilité des filles : les génisses issues de mères ayant reçu une supplémentation en fin de gestation au pâturage ont un taux de réussite à l'Insémination Artificielle (IA) supérieur à celles issues de mères non supplémentées (Martin et al 2007). Dans cet essai, la supplémentation a permis le maintien du poids et de la note d'état chez les animaux supplémentés, alors que l'on observe une perte de $29 \mathrm{~kg}$ et de 0,6 de note d'état chez les animaux non supplémentés en fin de gestation. Il est donc difficile de séparer l'effet du traitement de celui de l'évolution de la composition corporelle. Néanmoins, chez les filles, on n'observe pas de différence significative (poids, note d'état) en fonction du traitement initial des mères. A l'inverse, chez des animaux laitiers (Swali et Wathes 2007), le taux de réussite à la 1 ère IA est de $48 \%$ chez les génisses issues de vaches multipares contre $62 \%$ chez celles issues de vaches primipares $(\mathrm{P}<0,05)$. Après le 1 er vêlage, aucune différence n'est observée entre animaux. Selon ces auteurs, le retard de croissance intra-utérin, qui se traduit par un poids et un développement moins important à la naissance, entraîne des performances de croissance supérieures chez les issues de primipares, via un environnement endocrinien différent (GH, IGF1 notamment), ce qui aurait également un effet positif sur la fertilité.

Des analyses réalisées sur 640 animaux élevés sur le domaine INRA du Pin au Haras situé dans l'Orne de 1984 à 2002 confirment certaines des différences entre génisses issues de primipares et issues de multipares (poids à la naissance par exemple), mais elles n'ont pas démontré de différence sur la fertilité ultérieure des animaux (Charton 2008). Cependant, les conditions d'élevages, notamment l'âge au $1^{\text {er }}$ vêlage (24 et 36 mois), divergeaient entre ces études.

L'organogenèse utérine est assez bien connue et les principales étapes ont lieu au cours des 2 ème, 3 ème et 4 ème mois de gestation (Pavaux 1981). Les ovaires et l'utérus vont ensuite croître de manière importante. Chez une génisse Holstein, le poids de l'utérus est multiplié par 22,5 de la naissance à 10 mois d'âge, alors que son poids n'est multiplié que par 7 à 8 . En cours de gestation, 1'utérus de vache s'accroît en moyenne de $0,5 \mathrm{~kg}$ à $6-10 \mathrm{~kg}$ en fin de gestation, pour une évolution total du poids utérin de $0,8 \mathrm{~kg}$ à $40-80 \mathrm{~kg}$. Chez la génisse, la 1 ère gestation débute alors que l'animal n'a pas encore atteint sa maturité (environ $60 \%$ du poids adulte, Troccon 1996). Il peut ainsi exister une compétition entre tous les besoins précédemment évoqués, besoins qui peuvent aussi varier en fonction du numéro de lactation.

Il semble donc difficile de conclure sur l'existence ou non d'effets de la nutrition de la mère pendant la phase de gestation sur les performances de reproduction des filles, comme cela est observé dans d'autres espèces, notamment chez l'Homme (Chavatte-Palmer et al 2008, Gardner et al 2008). Il est ainsi sans doute nécessaire de poursuivre les investigations dans ce sens avant de pouvoir conclure de manière définitive.

\section{2 / Importance des condi- tions d'élevage avant se- vrage}

Au cours de la croissance, la composition corporelle des animaux varie de manière non linéaire et fluctue notamment en fonction des apports alimentaires. Pendant les premières semaines de vie, l'accroissement du poids vif est fortement corrélé à celui de ses tissus et organes constitutifs, de ses composants chimiques ou encore, de l'évolution du contenu digestif (Robelin 1986, figure 1). Tous ceux-ci ont un rythme de développement qui leur est propre et différent de celui des autres, ce qui conduit à des fluctuations importantes de composition corporelle au cours du temps. Mais outre les tissus, les différentes régions corporelles obéissent aussi à des rythmes de croissance différents. Ainsi, si la croissance de la tête est quasiment achevée à la naissance, celle du tronc est encore importante, alors que celle du bassin est faible (Huxley 1932).

$\mathrm{Au}$ cours des premiers mois qui suivent la naissance, les possibilités de croissance en tissus musculaires et osseux sont ainsi les plus importantes. Or, d'après une revue de Troccon et Petit (1989), il existe une très forte cor-

Figure 1. Evolution du gain des principaux constituants chez les bovins en cours de croissance (d'après Robelin 1986).

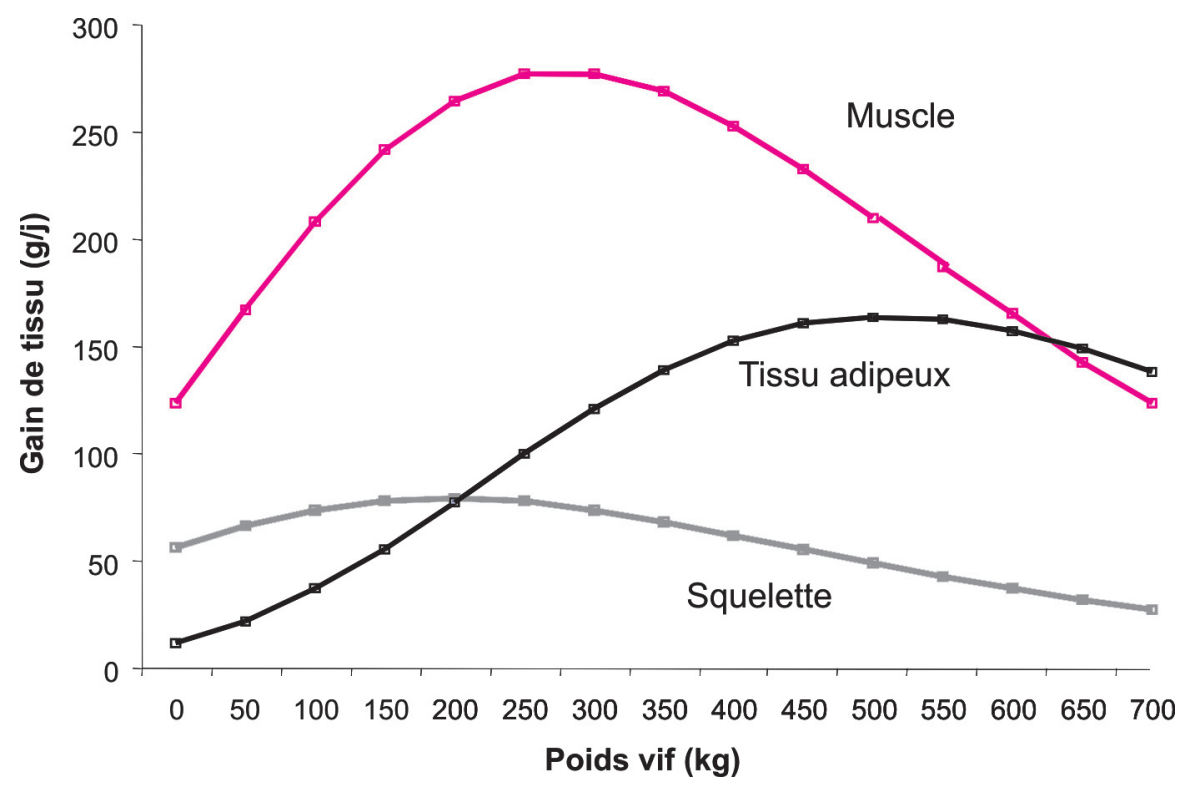


Tableau 1. Composition du colostrum et des laits en fonction du numéro de traite (d'après Foley et Otterby 1978).

\begin{tabular}{|l|c|c|c|c|}
\hline & \multicolumn{4}{|c|}{ Numéro de traite } \\
\cline { 2 - 5 } & $\mathbf{1}$ & $\mathbf{3}$ & $\mathbf{5}$ & $\mathbf{1 1}$ \\
\cline { 2 - 5 } & Colostrum & \multicolumn{2}{|c|}{ Lait de transition } & Lait entier \\
\hline Matière sèche, \% & 23,9 & 14,1 & 13,6 & 12,5 \\
Lipides, \% & 6,7 & 3,9 & 3,5 & 3,2 \\
Protéines, \% & 14,0 & 5,1 & 4,1 & 3,2 \\
dont anticorps & $6,0(2-23)$ & 2,4 & 0,1 & 0,09 \\
Lactose, \% & 2,7 & 4,4 & 4,7 & 4,9 \\
\hline
\end{tabular}

rélation entre les performances de croissance entre 0 et 6 mois d'âge et le gabarit de la vache adulte, corrélé à sa capacité d'ingestion. Selon ces auteurs, un retard de croissance dans le tout jeune âge est difficilement compensable par la suite. Il convient donc de maintenir une croissance élevée au cours de cette période, afin d'assurer une bonne stature des animaux.

\section{1 / Cas particulier du colos- trum}

Le colostrum est le premier aliment consommé par le jeune. Par sa richesse en anticorps et en lipides, il apporte à la fois une protection immunitaire au veau qui en est démuni à la naissance et une quantité d'énergie suffisante pour assurer sa survie initiale (thermorégulation, métabolisme de base...). Sa composition évolue rapidement (tableau 1). Au bout de $24 \mathrm{~h}$ de vie, les teneurs en protéines (dont les anticorps) et lipides ont déjà fortement chuté. De plus, la capacité d'absorption intestinale via les entérocytes de l'intestin grêle diminue rapidement et celle-ci est quasiment nulle au-delà de $24 \mathrm{~h}$ de vie (Weaver et al 2000). On observe également une très forte corrélation positive entre rapidité de la prise colostrale et teneur sérique en immunoglobulines (IgG), pour un même volume et une même concentration de colostrum. L'ingestion d'IgG est maximale durant les 4 premières heures de vie (Weaver et al 2000). Il est donc important pour la future génisse de consommer un colostrum de qualité et de quantité suffisantes dès les premières heures de vie. En effet, on observe une forte corrélation entre la teneur en IgG sériques et la survie du veau : plus cette teneur est élevée, plus les chances de survie augmentent (figure 2, d'après Wattiaux 1997). Pour une bonne protection immunitaire, un minimum de $10 \mathrm{mg}$ d'anticorps par $\mathrm{mL}$ dans le sang $24 \mathrm{~h}$ après naissance est à rechercher. Ceci correspond à 2 litres de colostrum après naissance, puis 2 litres 6 à $12 \mathrm{~h}$ plus tard. Le volume doit néanmoins être adapté suivant le poids vif de l'animal, c'est-àdire, réduit chez des animaux de faible poids $(<35 \mathrm{~kg})$ ou augmenté chez les individus les plus lourds ( $>45 \mathrm{~kg})$. Le colostrum, riche en anticorps, doit aussi apporter de l'énergie en quantité suffisante. En effet, selon des observations réalisées chez le porc, la quantité donnée peut être suffisante pour assurer une bonne protection immunitaire, mais elle est parfois insuffisante en apports énergétiques, ce qui peut conduire à la mort de l'animal (Le Cozler et Le Dividich 2004). Chez le veau, une telle situation est toutefois très rarement rencontrée.

Les facteurs de variation, tant du point de vue de la qualité que de la quantité, sont assez bien connus (Foley et Otterby 1978). Ils dépendent notamment de la durée du tarissement et de celle de la gestation (moins d'anticorps en cas de tarissement inférieur à 4 semaines ou en cas de fin de gestation prématurée), de la race (teneur en anticorps plus faible chez les vaches Holstein), de l'âge de la vache (teneur en anticorps plus élevée chez les animaux âgés par rapport aux primipares), etc. Dans ce dernier cas, si la qualité immune du colostrum de primipares a parfois été mise en doute, plusieurs études montrent une absence de différence entre les concentrations en $\operatorname{IgG}$ entre des laits de primipares et de multipares (Weaver et al 2000). Si une sélection de colostrum devait toutefois être effectuée sur la base de la parité de l'animal,

Figure 2. Importance du délai naissance-prise colostrale et de la quantité ingérée par repas sur l'acquisition immunitaire et la survie des veaux (d'après Wattiaux 1997).

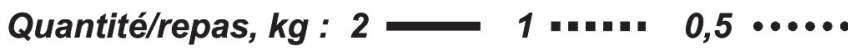

Mortalité des veaux

ation sérique en IgG, $\mathrm{mg} / \mathrm{mL}$

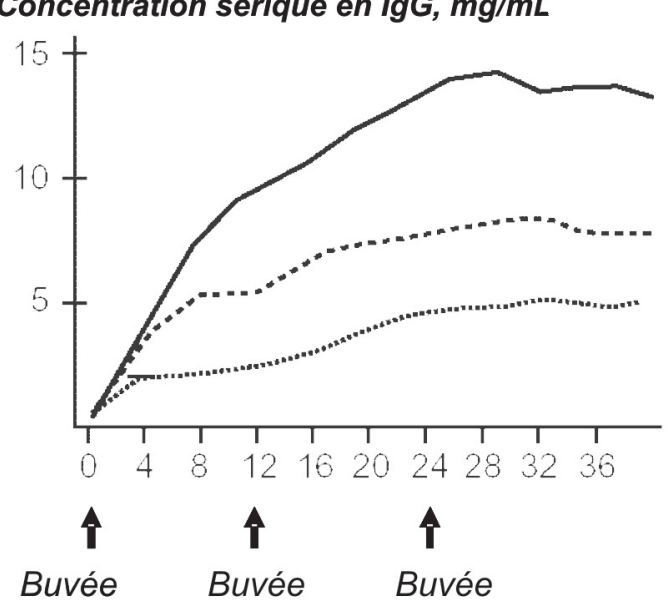

Heures après naissance

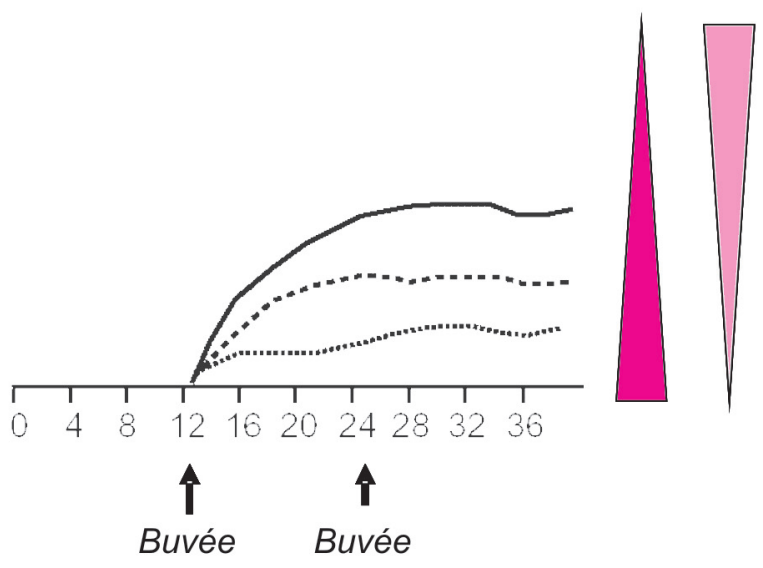

Heures après naissance 
ces auteurs recommandent d'utiliser des colostrums issus de vaches en troisième lactation. En ce qui concerne les races, Muller et Ellinger (1981) ont montré que les colostrums de vaches Holstein avaient une concentration en IgG plus faibles que ceux issus de vaches Jersiaise (respectivement 90,4 vs 55,9 g/L). Enfin, lorsque la durée de tarissement est 0,10 ou 60 jours, la concentration en immunoglobulines dans la colostrum est de 33, 63 et 72 $\mathrm{g} / \mathrm{L}$, ce qui illustre bien l'importance de la durée de cette période (Rémond et al 1997).

D'autres facteurs, comme certaines vaccinations et l'exposition antérieure à divers pathogènes, ont également un effet plus ou moins important (Weaver et al 2000). Gulliksen et al (2008) ont aussi démontré que la qualité du colostrum pouvait varier selon les mois de vêlage, avec des teneurs en IgG plus faibles chez les vaches vêlant lors des mois de décembre, janvier et février : moins de $50 \mathrm{~g} / \mathrm{L}$ au cours de ces mois contre des valeurs supérieures à cette valeur seuil lors des autres mois. Néanmoins, Pritchett et al (1991) n'ont pas observé cette évolution, mais les variations (température et régimes alimentaires notamment) étaient plus faibles dans cette étude. Enfin, des travaux de Dardillat et al (1978) ont montré qu'il existait une certaine répétabilité de la qualité du colostrum pour une même vache au cours des différentes lactations. En effet, la répétabilité de la teneur en immunoglobulines du colostrum a été calculée comme étant voisine de 0,20 . Lorsque le modèle utilisé pour ces calculs prenait en compte l'effet année, cette valeur était proche de 0,41 . Ces auteurs ont aussi trouvé un coefficient de corrélation variant de 0,23 à 0,29 , suivant le modèle utilisé, entre les concentrations en IgG produites par les mères et celles des filles, sur une base néanmoins limitée de 32 vaches et leur descendance. Ils ont ainsi conclu qu'il devait exister une certaine héritabilité de la teneur en IgG du colostrum.

\section{2 / Consommation de lait et d'aliment solide}

Pendant les premières semaines de vie, le lait apporte l'essentiel des nutriments et couvre les dépenses de l'animal. Le veau peut recevoir un aliment d'allaitement reconstitué ou du lait entier, en 1 ou 2 repas par jour (tableau 2). Dans le cas du lait entier, on peut tout à fait valoriser le lait non commercialisable mais sain (les laits des 14 premières traites ou encore les laits riches en leucocytes mais avec moins d'un million de cellules/mL). Le lait issu de vaches présentant des mammites cliniques, plus pauvre qu'un lait normal, est à proscrire (Troccon 1984). Il faut aussi surveiller la teneur en matière grasse du lait. Les quantités de lait entier distribuées doivent être réduites de $0,5 \mathrm{~L} / 4$ points de taux butyreux au-dessus de $40 \mathrm{~g} / \mathrm{kg}$ (Agabriel et Meschy 2007). En effet, selon Troccon (1984), pour de bonnes efficacité et utilisation du lait consommé, le jeune veau ne pourrait absorber plus de $400 \mathrm{~g}$ de matières grasses par $100 \mathrm{~kg}$ de poids vif. Quelques précautions supplémentaires doivent être prises avec des aliments d'allaitement permettant d'obtenir un lait reconstitué. La dilution doit notamment tenir compte des teneurs en matières grasse et protéique. Selon Agabriel et Meschy (2007), on peut fournir un $\mathrm{kg}$ de lait au veau, à partir de 220 g d'aliment d'allaitement et 780 g d'eau, ou à partir de $750 \mathrm{~g}$ de lait entier, complété de $125 \mathrm{~g}$ d'aliment d'allaitement préalablement dilués dans $125 \mathrm{~g}$ d'eau, si on a recours à l'utilisation d'un mélange (lait entier + lait en poudre).

La comparaison de plans simples (1 repas par jour, quantité constante tout au long de la période lactée) ou complexes (via l'utilisation de DAL) montre peu de différences, en termes de performances de croissance des animaux (Brunschwig et al 2005). Les techniques utilisant des ferments lactiques, très utilisées en NouvelleZélande et en Irlande, se sont développées depuis plusieurs années en France. Il existe néanmoins peu de données précises sur leurs effets sur le plan zootechnique. Cependant, de faibles investissements nécessaires à leurs mises en place, un temps de préparation et de distribution réduits, ainsi qu'une distri- bution à température ambiante rendent ces techniques attrayantes et faciles d'utilisation (Porhiel 2008a). Le plan lacté avec un lait fermenté ou «yoghourt» est similaire à un plan classique d'allaitement (colostrum au départ, $8 \mathrm{~L} / \mathrm{veau} / \mathrm{j}$ jusqu'à l'âge de 7 semaines, puis diminution progressive). Il faut toutefois noter que le lait fermenté n'apparaît pas dans la liste positive des aliments d'allaitement reconnus chez le veau, c'est-à-dire, dans la liste des aliments reconnus et autorisés chez le veau.

Selon certaines études, il existerait une différence entre lait de remplacement et lait entier : meilleure croissance, développement corporel plus important, puberté plus précoce et au final, tendance à produire plus de lait chez des génisses nourries au lait entier par rapport à celles recevant un lait de remplacement pendant les 42 premiers jours de vie (Bar-Peled et al 1997). La composition exacte du lait de remplacement est néanmoins peu précise dans cet essai. Or, la composition des aliments d'allaitement, notamment lorsque la poudre de lait écrémé est remplacée par d'autres sources de protéines, a un impact sur les performances zootechniques, mais aussi sur les risques sanitaires (Troccon et Toullec 1989). Les protéines de substitution sont en effet incoagulables dans la caillette, ce qui entraîne une évacuation plus rapide des protéines et des lipides. Outre des risques d'intolérance et de perturbation des secrétions digestives, les risques de diarrhées et de mortalité sont accrus. Et lorsque le taux de substitution atteint 25 à $50 \%$ suivant les protéines et les traitements technologiques, le gain de poids vif relatif des veaux de moins de 6 semaines chute fortement (5 à 25\%) par rapport aux animaux témoins (Troccon et Toullec 1989).

Tableau 2. Quelques exemples de plans d'allaitement de veaux sevrés à 8 semaines et réalisant un GMQ de $900 \mathrm{~g} / \mathrm{j}$ (kg/j). D'après Agabriel et Meschy (2007).

\begin{tabular}{|c|c|c|c|c|c|c|c|c|c|}
\hline \multirow{2}{*}{ Alimentation } & \multicolumn{9}{|c|}{ Semaine } \\
\hline & 1 & 2 & 3 & 4 & 5 & 6 & 7 & 8 & 9 \\
\hline $\begin{array}{l}\text { Lait entier* ou lait de remplacement, } \\
2 \text { repas } / \mathrm{j}(1 \mathrm{~kg}=130 \mathrm{~g} \text { aliment allaitement } \\
+870 \mathrm{~g} \text { eau })\end{array}$ & 6 & 8 & 8 & 8 & 8 & 8 & 6 & 3 & 0 \\
\hline $\begin{array}{l}\text { Lait de remplacement, } 1 \text { repas } / \mathrm{j} \\
(1 \mathrm{~kg}=220 \mathrm{~g} \text { aliment allaitement } \\
+780 \mathrm{~g} \text { eau ou } 750 \mathrm{~g} \text { lait entier } \\
+125 \mathrm{~g} \text { aliment allaitement } \\
+125 \mathrm{~g} \mathrm{eau})\end{array}$ & 3 & 4 & 5 & 5 & 5 & 5 & 3,5 & 2 & 0 \\
\hline
\end{tabular}

* suivant la teneur en matières grasses, réduire de 0,5 L/ 4 points de taux butyreux au-dessus de $40 \mathrm{~g} / \mathrm{kg}$. 
La mise à disposition d'aliments solides dès les premières semaines de vie a un effet positif sur le développement du rumen et sa colonisation par les bactéries. Les quantités de concentrés et de fourrages apportées et/ou consommées varient en fonction de leurs qualités (INRA 2007). Ces aliments, concentrés et fourrages, ont un effet bénéfique sur la croissance et le développement du tube digestif, respectivement. Selon Lyford (1988), les aliments concentrés fermentescibles auraient un effet positif sur la croissance papillaire alors que le développement de la musculeuse des préestomacs est influencé par des aliments de lest. Il faut donc veiller à apporter suffisamment d'énergie pour assurer un niveau de croissance élevé, tout en veillant à ne pas pénaliser le développement du rumen.

Enfin, pour décider de sevrer ou non une génisse, le doublement du poids de naissance est un repère à minima, ce qui est le cas généralement lorsque le veau est âgé de 7 à 8 semaines environ (Troccon 1989). Il est souhaitable que le veau consomme déjà $2 \mathrm{~kg} \mathrm{MS} / \mathrm{j}$ d'aliments solides pour le réaliser, sous peine de voir les performances zootechniques et sanitaires se détériorer sérieusement après ce sevrage. Néanmoins, comme lors de la succession des périodes d'hivernage et de pâturage, des possibilités de rattrapage existent suite au retard dû à une alimentation lactée insuffisante, mais cela nécessite la distribution d'une quantité de concentrés journalière plus importante que les $2 \mathrm{~kg}$ recommandés jusqu'à l'âge de 4 mois (Troccon 1989). Lorsque le sevrage est précoce et que la quantité d'allaitement distribuée depuis la naissance a été faible (moins de 30 $\mathrm{kg}$ ), Troccon (1989) recommande d'ailleurs de distribuer plus de $3 \mathrm{~kg}$ de concentrés après sevrage.

\section{3 / Elevage du sevrage à la mise à la mise à la repro- duction}

D'une manière générale, une croissance trop élevée avant la puberté a un effet négatif sur la production de lait (via ses effets sur le développement mammaire), mais elle accélère l'apparition de la puberté. Cet effet plus ou moins négatif sur la production de lait, notamment en première lactation, dépend principalement de la période d'application du traitement (Le Cozler et al 2009a).

Figure 3. Influence du niveau de croissance de la naissance à la puberté sur l'apparition du premier oestrus (Troccon et Petit 1989).

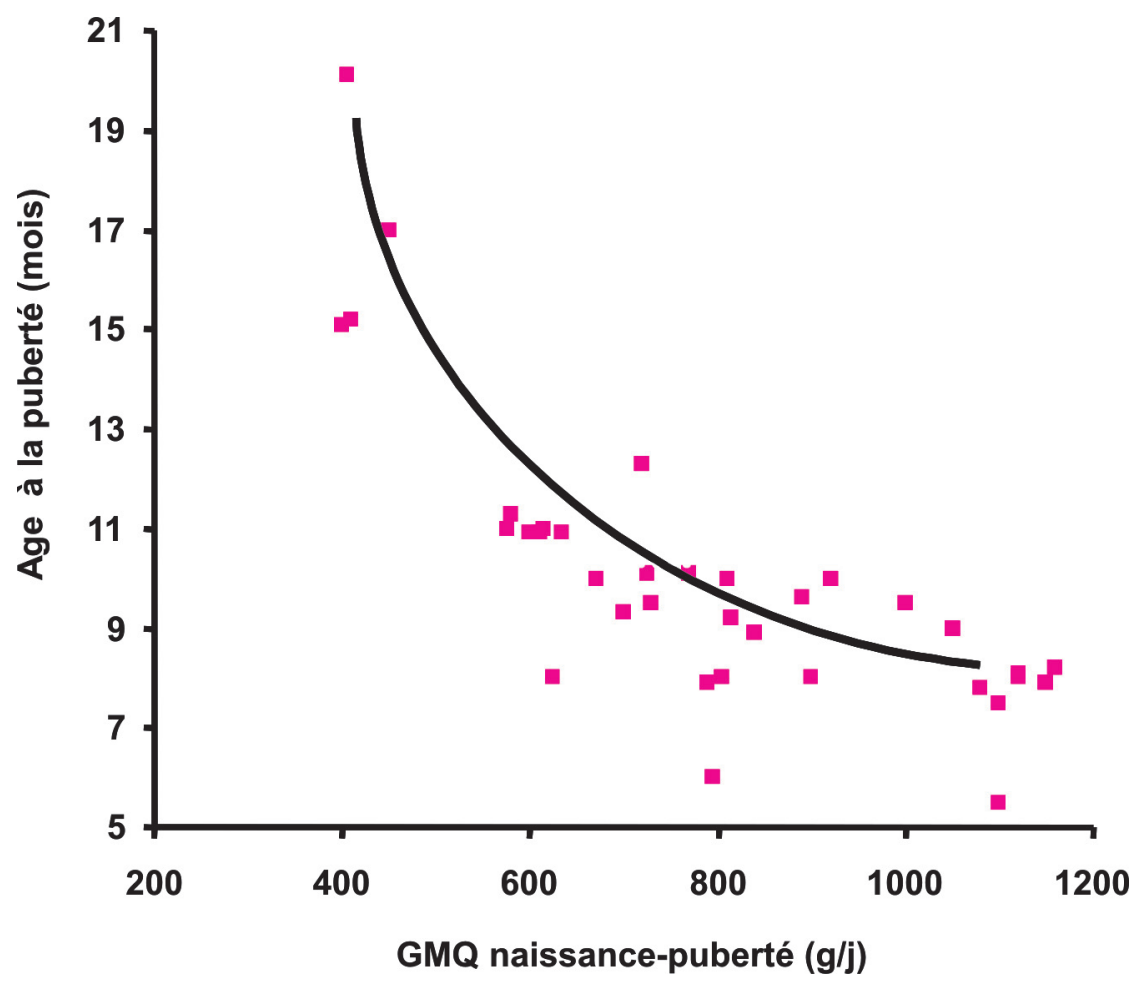

\section{1 / Acquisition de la puberté}

L'apparition de la puberté est fortement influencée par le développement (40 à 50\% du poids, Troccon 1996, Le Cozler et al 2009a) et donc, par la vitesse de croissance de l'animal : plus elle est élevée, plus l'apparition de la puberté est précoce (figure 3, Troccon et Petit 1989). Lorsque la croissance atteint 850-900 g/j, la puberté est obtenue à 9-10 mois chez la plupart des génisses de race Holstein (Wattiaux 1997). Comme l'illustre la figure 3, il existe une grande variabilité de l'âge à la puberté autour des valeurs «cibles», même pour une croissance considérée. Néanmoins, le seuil de 9 mois (270 j) est parfois diminué, lorsque le niveau de croissance est supérieur à $900 \mathrm{~g} / \mathrm{j}$ : il passe ainsi de 273 à $251 \mathrm{j}$ lorsque le Gain Moyen Quotidien (GMQ) passe de 909 à $978 \mathrm{~g} / \mathrm{j}$ depuis la naissance (Le Cozler et al 2009a). Inversement, lorsque le GMQ est faible $(<350 \mathrm{~g} / \mathrm{j})$, la puberté est atteinte entre 18 et 20 mois d'âge.

Une puberté précoce peut être induite chez une majorité de génisses grâce à un sevrage précoce et une ration à haut niveau en concentrés (Le Cozler et al 2009a). Ainsi, chez des génisses allaitantes, augmenter les apports énergétiques de la ration chez les génisses sevrées précocement (112 j, $150 \mathrm{~kg})$, en leur donnant une ration à haut $(60 \%$ de maïs dans la ration) ou bas (30\% de maïs dans la ration) niveau en concentrés de 126 à 196 jours, abaisse l'âge à la puberté (moins de 290 j contre plus de 300 j) (Gasser et al 2006). Lorsque la ration est enrichie ou appauvrie en énergie au-delà de 196 jours, aucune différence n'est observée entre traitements. Par ailleurs, des génisses rationnées, puis nourries à volonté peu de temps avant la puberté attendue, présentent leurs premières chaleurs 22 jours avant les génisses témoins (Peri et al 1993). Une des hypothèses avancées est que pendant leur courte période de compensation, et en raison à la fois d'un métabolisme basal réduit et donc d'un surplus d'énergie et de protéines lors de la réalimentation (Hornick et al 2000), elles pourraient déposer davantage de lipides, favorable au déclenchement de la puberté, comme cela a été rapporté dans d'autres espèces (Frisch 1984). Néanmoins, les mécanismes liés à la croissance compensatrice ne sont pas totalement bien connus, notamment en ce qui concerne la nature et le moment précis des gains (Hornick et al 2000, Hoch et al 2003). Cette acquisition de la maturité sexuelle semble aussi indépendante du régime utilisé (Le Cozler et al 2009a) et fortement liée au GMQ en période prépubertaire. Au final, l'acquisition de la puberté est donc plus fortement liée au développement corporel (poids, niveau des réserves corporelles notamment) de l'animal qu'à son âge. 
Figure 4. Effet de l'âge à l'IA sur la fertilité (\% de réussite à l'IA) des génisses (d'après Barbat et al 2007).

\section{$\%$ réussite IA, par rapport à la moyenne observée}

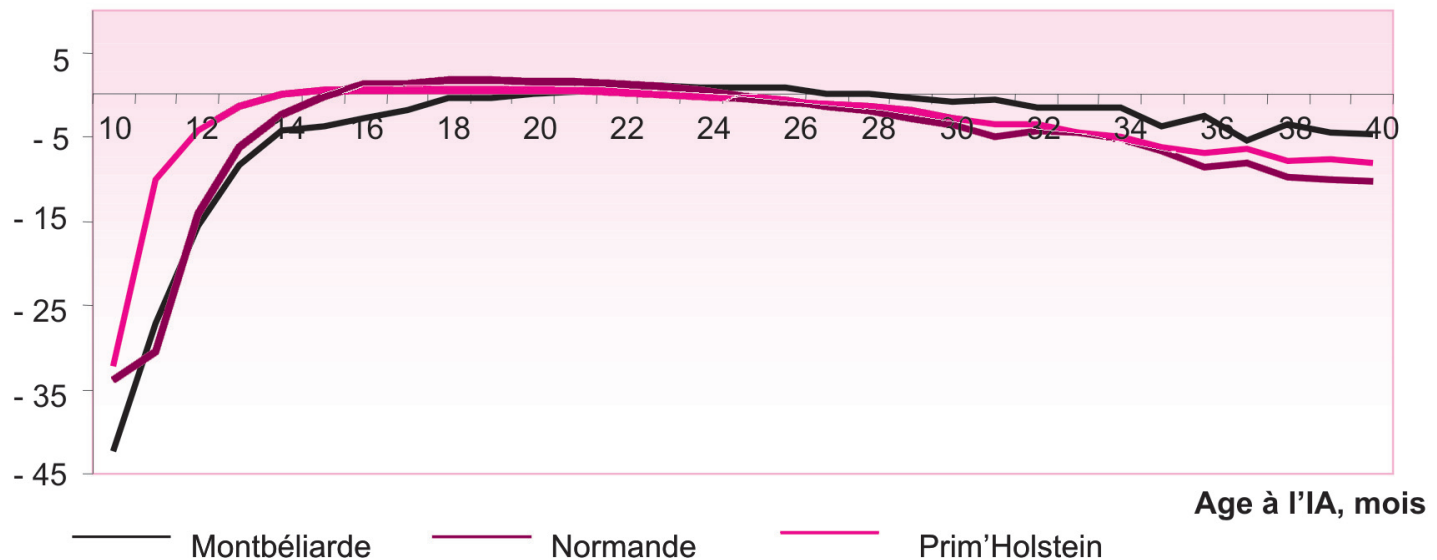

Il existe néanmoins des différences entre races. Les génisses de races laitières à croissance rapide (du type Holstein) et certaines autres (par exemple de type Jersiaise) présentent une précocité sexuelle plus importante que les races à croissance plus lente (du type Normande, Montbéliarde ou Tarentaise), et a fortiori, que les races allaitantes (Gauthier et al 1986, D'Hour et al 1995, Troccon 1996). Il s'agit d'un paramètre important à prendre en compte lorsque l'on désire faire des vêlages groupés, avec un âge au $1^{\mathrm{er}}$ vêlage précoce (24 mois). Cette différence de précocité sexuelle entre races se retrouve non seulement au niveau de la puberté, mais aussi au niveau des taux de fertilité à la 1 ère IA. On observe que la mise à la reproduction en dessous de 15 mois apparaît tout à fait réaliste et ne pénalise pas ce critère chez ces races précoces (figure 4, Barbat et al 2007), même s'il ne semble pas y avoir unanimité sur le sujet. Mais si Philipot (2008) observe une légère baisse de la fertilité lorsque l'âge à l'IA diminue en dessous de 15 mois, il reconnait que tout concorde pour dire qu'inséminer à 15 mois ne diminue pas la fertilité et que ce sont sans doute les conditions d'élevage qui peuvent expliquer cette évolution. Dans les conditions de vêlages groupés, la variabilité des croissances individuelles au sein d'un lot, liée au potentiel de chaque animal ou tout simplement à sa date de naissance lors de la saison de vêlage, ne permet pas toujours d'atteindre les objectifs de poids et de développement à l'âge voulu. Il s'agit alors pour chaque éleveur de réfléchir aux différentes options possibles, en fonction de son système : mettre néanmoins à la reproduction, retarder celle-ci d'un an, accélérer la croissance des animaux nés tardivement en saison, les réformer plus ou moins précocement.

\section{2 / Cyclicité et mise à la repro- duction}

Dans de nombreuses espèces animales, il existe une relation positive entre fertilité et numéro d'oestrus (ou chaleur détectée) après la puberté, indépendamment de la vitesse de croissance initiale (Le Cozler et al 1999). Attendre une ou plusieurs chaleurs après l'apparition de la puberté se traduit par une amélioration du taux de fertilité. Lorsque la croissance est lente, la fertilité est améliorée si les animaux sont inséminés à la $3^{\text {ème }}$ ou 4 ème chaleur plutôt qu'à la puberté (Byerley et al 1987, chez des races allaitantes par exemple). Chez les génisses de race Holstein où la puberté est précoce (entre 6 et 9 mois d'âge) et la croissance importante, retarder audelà de 26 mois la mise à la reproduc- tion (ex. : vêlage au-delà de 3 ans) est peu adapté, la fertilité baissant de $10 \%$ au-delà de 2 ans (figure 4, d'après Barbat et al 2007). De même, cette analyse montre un effet fortement pénalisant pour les IA réalisées sur des jeunes génisses de race Montbéliarde. Dans le cas d'un vêlage tardif (3 ans), Troccon et al (1997) ont montré l'intérêt d'une croissance modérée (de l'ordre de $550 \mathrm{~g} / \mathrm{j}$ ) avant 14 mois d'âge pour l'obtention de meilleurs taux de fertilité par rapport à une croissance rapide. L'âge au vêlage élevé semble donc mieux adapté aux animaux à croissance lente et doit donc être adapté à chaque système.

Dans les cas extrêmes où l'alimentation est franchement insuffisante, la cyclicité peut même disparaître (figure 5) : chez des génisses de race Holstein, le pourcentage de génisses cyclées diminue fortement lorsque la

Figure 5. Effet du niveau de croissance après la puberté sur le maintien de la cyclicité de génisses Holstein (d'après synthèse bibliographique, Disenhaus et Troccon communication personnelle).

\section{$\%$ génisses cycliques}

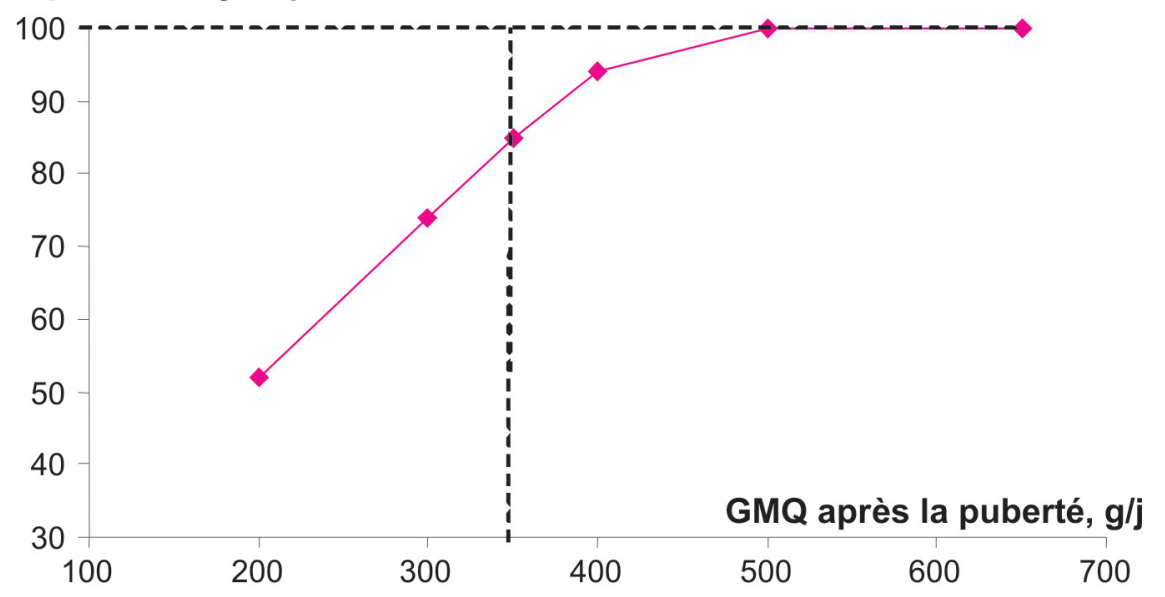


croissance après puberté est inférieure à $400 \mathrm{~g} / \mathrm{j}$ (Disenhaus et Troccon communication personnelle). Un déficit énergétique ou protéique sévère se solde par l'arrêt des cycles oestriens chez la génisse (Kaur et Arora 1995). Cette perte est néanmoins réversible lorsque l'animal est de nouveau alimenté de manière suffisante. Selon Troccon (1996), le système IGF1 (Insulin like Growth Factor 1) aurait un rôle clé de médiateur dans les effets de l'alimentation sur la reproduction des ruminants. Une altération de la teneur en IGF1 pourrait se traduire par un effet négatif sur les niveaux circulants et l'amplitude des pics de LH, ainsi que sur ses récepteurs ovariens, se traduisant par un effet direct sur la sécrétion ovarienne de stéroïdes et un arrêt de cyclicité.

Enfin, des retards de croissance dus à la présence de parasites entraînent une baisse de gain de poids et des retards dans l'apparition de la puberté (LacauMendigo et al 2000). Dans des cas extrêmes, on pourrait supposer qu'ils puissent avoir des effets proches d'une restriction sévère. L'utilisation d'anthelminthiques au cours de la croissance permet d'éviter ces problèmes et souligne bien l'importance d'une prophylaxie réfléchie et adaptée chez les génisses, notamment autour du pâturage.

La fertilité des nullipares peut-être considérée normale à partir de valeurs d'un taux de réussite à la 1 ère IA supérieur ou égal à $60 \%$. Ces valeurs sont généralement observées pour des gains de poids vif variant de 340 à $680 \mathrm{~g} / \mathrm{j}$ au moment de la mise à reproduction (23 mois et $380 \mathrm{~g} / \mathrm{j}$ en cas de vêlage à trois ans ou 14 mois et $590 \mathrm{~g} / \mathrm{j}$ lors de vêlage à deux ans). Un gain de poids vif compris entre 700 et $800 \mathrm{~g} / \mathrm{j}$ à la mise à reproduction permet de concilier un vêlage à 24 mois et une fertilité optimale. Pour un vêlage tardif, cet optimum est de $500 \mathrm{~g} / \mathrm{j}$ (Disenhaus 1991). Une fertilité élevée dépend de l'état corporel (note de 3 à 3,2 suivant la grille développée par Le Mat et al 1994), mais aussi du gain de poids autour de la période de l'insémination (IA) (Pierre 2003).

\section{4 / Phase de production (de la mise à la reproduction à la réforme)}

Au début de sa carrière comme reproducteur, la future vache laitière doit faire face à des besoins de croissance, puis de croissance et de maintien de la gestation, et enfin, en début de première lactation, de production de lait, de croissance et de reproduction ultérieure.

\section{1 / Gestation et premier vêlage}

L'objectif d'élevage des génisses est non seulement d'assurer le développement mammaire pour un bon potentiel laitier, mais aussi, une carrière satisfaisante. Pour cela, il est nécessaire d'avoir des génisses en état optimal au premier vêlage : leur conduite pendant la gestation permet d'avoir de tels animaux si ce n'était pas le cas avant. En effet, la reprise rapide de cyclicité lors de la première lactation est généralement liée à la note au vêlage et à la perte d'état qui suit. Selon Disenhaus et al (2005), les analyses effectuées dans le troupeau expérimental INRA de Mejusseaume (35) montre que c'est même la perte d'état qui est le premier facteur explicatif du succès à l'IA1 chez la primipare, alors que chez la multipare, sans renier pour autant son importance, il apparaît comme facteur associé plutôt que facteur en tant que tel. Les deux anomalies de reprises de cyclicité les plus fréquemment rencontrées sont les phases lutéales prolongées et l'inactivité prolongée (Grimard et Disenhaus 2005). Des animaux maigres et/ou ayant une perte importante d'état (>1,5 par exemple), ce qui traduit un déficit énergétique important en début de lactation, ont généralement comme conséquences des retards de venue en chaleur et des taux de conception à l'IA plus faibles (Lopez-Gatius et al 2003, Grimard et Disenhaus 2005, Disenhaus et al 2005, Chagas et al 2006). Ceci se traduit aussi au final par un nombre plus important de vaches non gestantes au bout de 150 ou 200 jours de lactation (Walsh et al 2007). Les retards de venue en chaleur pourraient s'expliquer par un effet direct du déficit énergétique sur la pulsatilité de l'Hormone Lutéinisante (LH) (Grimard et Disenhaus 2005). Ce déficit est généralement accentué chez les vaches fortement productrices et celles qui ont des capacités d'ingestion moindre, comme les primipares.

Globalement, les animaux trop maigres ou trop gras ont davantage de problèmes sanitaires autour du vêlage (Philipson 1976, Disenhaus et al 1986). Ces problèmes, généralement de dystocies et/ou de rétention placentaires, sont minimisés avec une note d'état corporel variant entre 2,5 et 3,5 au moment du vêlage. En effet, ces auteurs observent, dans des essais réalisés dans deux pays et à deux moments différents, un pourcentage d'environ 15 à $16 \%$ de dystocies, pour une note d'état au vêlage voisine de 3 . Lorsque cette note diminue à 1 ou augmente à 4,5-5, le pourcentage de dystocies avoisine les $25 \%$. Les difficultés de parturition chez la primipare induisent des problèmes de rétention placentaire, des mammites et au final, aboutissent à des taux de réformes élevés (Erb et al 1985, Augeard et al 1986). Des études menées par Ford et Park (2001) mettent en avant néanmoins l'importance de la conduite de la gestation des génisses et notamment, l'importance du dernier tiers. Selon ces auteurs, certains effets négatifs apparus lors de la phase prépubère (croissance pas assez trop élevée) pourraient être en partie annulés par une modification des conditions d'alimentation (et donc de croissance) au cours de la gestation. L'effet d'une restriction sévère en phase prépubère pourrait alors être remis en cause, car selon eux, dans la plupart des essais publiés sur le sujet, il pourrait exister une confusion entre les modèles utilisés (contrôlés ou pas), le type d'essai (restriction sur l'énergie ou la quantité totale), l'âge des animaux et donc, le statut hormonal (prépubère, pubère, gestant...), la durée des essais qui peut couvrir plusieurs de ces phases. Ceci pourrait expliquer aussi pourquoi dans la littérature, il n'existe pas de consensus entre auteurs sur l'impact de l'alimentation avant la puberté sur les performances laitières ultérieures (Le Cozler et al 2008). Toutefois, ils ne remettent pas en cause l'effet négatif d'un apport énergétique élevé sur le développement de l'animal et de sa mamelle, mais ils estiment qu'une bonne adéquation entre des apports énergétiques élevés et le statut hormonal particulier des animaux pourrait améliorer considérablement leurs performances. Ceci pourrait être obtenu grâce à une croissance discontinue, alternant des phases de croissances rapides et des phases lentes. La fin de gestation, lorsque la croissance tissulaire est maximale et que la lactation se prépare, serait alors aussi cruciale, voire plus, que toutes les autres phases de la croissance. Ces travaux ont toutefois été menés sur un nombre limité d'animaux et des études complémentaires nécessitent d'être réalisées pour valider cette hypothèse.

\section{2 / Carrière des animaux}

Les données sur les carrières entières sont peu nombreuses et des observations anciennes (Reid et al 1964) ten- 
Figure 6. Evolution du poids vif de trois lots de génisses vêlant à 36 mois en fonction du profil de croissance autour de l'IA et résultats à la mise à la reproduction (d'après Peccate et al 2006).

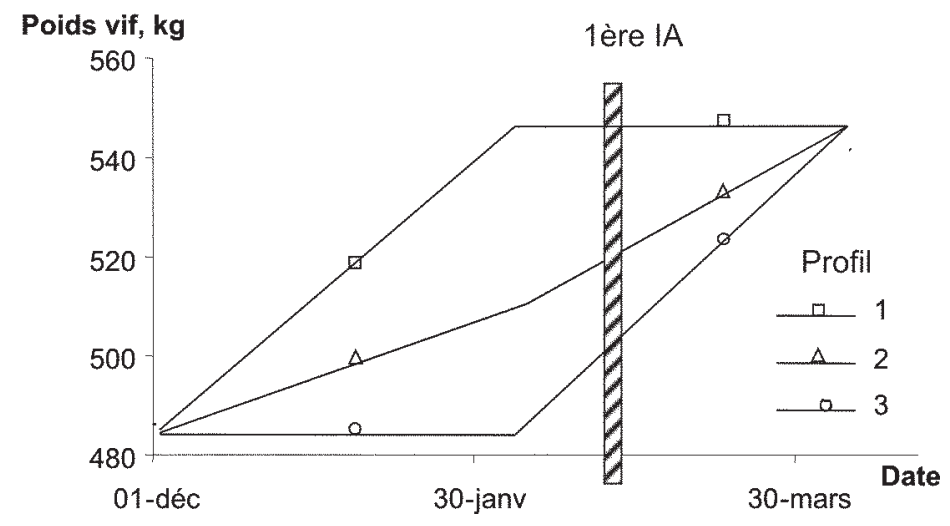

\begin{tabular}{|l|c|c|c|}
\hline Profil & $\mathbf{1}$ & $\mathbf{2}$ & $\mathbf{3}$ \\
\hline Effectif & 70 & 69 & 70 \\
\hline Phase 1 (70 j) & & & \\
PV début, kg & 485 & 486 & 486 \\
PV fin, kg & $543^{\mathrm{a}}$ & $510^{\mathrm{b}}$ & $485^{\mathrm{C}}$ \\
\hline Phase 2 (60 j) & & & \\
PV fin, kg & 548 & 543 & 542 \\
\hline \% réussite IA1 ${ }^{1}$ & 64,3 & 58,0 & 61,4 \\
Taux gestation, \% & 91,4 & 91,3 & 92,9 \\
\hline
\end{tabular}

Au sein d'une même ligne, deux chiffres avec une lettre différente diffèrent à $P<0,05$ 1 IA1: première insémination

dent à démontrer qu'il y a peu d'effet de l'intensité de croissance sur la longévité des animaux, estimée à partir du nombre de lactations réalisées avant la réforme. Les auteurs notaient aussi un impact économique négatif d'une croissance trop élevée. Dans cet essai, la croissance prépubère estimée des animaux du lot qui avaient les performances satisfaisantes était de l'ordre de 600-650 g/j. Néanmoins, les races ont évolué et les valeurs de croissances optimales aussi. Selon Hohenboken et al (1995), dans les années 80, l'optimum de croissance avant puberté sur la production de lait chez la primipare était de $600-700 \mathrm{~g} / \mathrm{j}$. Ces auteurs l'ont estimé à 800-900 g/j actuellement et sans doute supérieur à $1000 \mathrm{~g} / \mathrm{j}$ dans quelques années. Il n'est donc pas surprenant d'observer des divergences dans les valeurs observées entre les auteurs (Le Cozler et al 2008). Les objectifs de croissance et la recherche de valeurs optimales doivent donc être réfléchis au cas par cas, adaptés à chaque élevage et à sa génétique propre.

L'hypothèse qu'une croissance très élevée (plus de $1000 \mathrm{~g} / \mathrm{j}$ ) avant la puberté soit non pénalisante par la suite semble donc plausible, mais son impact dépendrait à la fois de la période pendant laquelle elle se déroule, sa durée, la nature et la quantité des régimes utilisés. Ceci semble conforter les propositions de Ford et Park (2001) (voir précédemment). Ainsi, même avec des croissances très élevées autour de la puberté, la longévité des animaux peut être bonne. En comparant deux groupes d'animaux ayant un GMQ proche de $900 \mathrm{~g} / \mathrm{j}$ entre 4 et 12 mois d'âge, Le Cozler et al (2009a) ont montré que la nature du régime (herbe vs maïs + concentré) pouvait influencer la survie des animaux (meilleure chez les animaux nourris à l'herbe de 4 à 12 mois d'âge). Mais des animaux nourris au maïs sans complément et ayant une croissance de $750 \mathrm{~g} / \mathrm{j}$ obtiennent des performances équivalentes en terme de longévité. Les performances de lactation n'étaient pas altérées. Il paraît alors envisageable d'avoir des croissances élevées avant puberté, mais devant être adaptées en fonction des élevages, des types d'animaux (races) de la nature du régime fourrager et des possibilités de croissance par stade physiologique.

\section{5 / Pour une meilleure mâ̂- trise de la fertilité}

\section{1 / Programme alimentaire autour de la première IA (et flushing)}

L'effet de l'alimentation (négatif ou positif) sur la fertilité des génisses lors de périodes assez longues autour de l'IA est parfois évoqué. Dans un essai mené sur des génisses Holstein et Normande pesant $485 \mathrm{~kg}$ à l'entrée en bâtiment et pour un objectif de vêlage à 3 ans, trois programmes alimentaires ont été testés sur 130 jours environ (70 génisses par lot). L'objectif de cette expérimentation était d'avoir des poids identiques à la mise à l'herbe (544 kg), mais ceux-ci étaient donc différents au moment de l'IA (figure 6, Peccatte et al 2006). Au final, les génisses Holstein ont présenté un taux de réussite plus faible en 1ère IA que les génisses Normande (56 vs 68\%), avec néanmoins un taux de gestation similaire (94 et $89 \%$, respectivement). Cet effet de la race se retrouve également chez l'adulte et les différences entre animaux de race Holstein et Normande sont bien connues (Barbat et al 2005). Chez ces génisses vêlant à 3 ans, l'application de séquences d'alimentation différentes autour de la période de reproduction n'a pas eu d'effet sur la fertilité des génisses. Des résultats similaires ont été obtenus sur des génisses de race Charolaise (Dozias et al 2006). Ces observations méritent sans doute d'être également réalisées dans le cas d'un vêlage à deux ans, avec une étude sur l'intérêt de manipuler l'apport énergétique lors de la période hivernale, avant insémination, c'est-à-dire entre 12 et 15 mois d'âge.

Dans beaucoup d'espèces animales, l'augmentation de la quantité d'énergie allouée avant l'ovulation sur une courte période, appelée «flushing», a démontré un réel intérêt. Chez la génisse laitière, des travaux de Troccon et Petit (1989) ont montré que chez des animaux en mauvais état (note d'engraissement d'état $<2$ ), cette pratique qui consistait en un apport supplémentaire d'énergie $(+2 \mathrm{UFL} / \mathrm{j})$ pendant les 2 ou 3 semaines qui précèdent et suivent l'IA permettait une amélioration du taux de fécondation. Chez les animaux où l'état est encore moins bon, cette pratique n'a pas eu de réel bénéfice : la cyclicité a peut être déjà été perdue, voire même n'a jamais été acquise. Chez des animaux en bon ou très bon état (note $>3$ ), cette technique du flushing ne présente pas d'intérêt et peut même être pénalisante sur les performances de reproduction ultérieures.

\section{2 / L'utilisation des hormones exogènes}

La bonne connaissance des mécanismes physiologiques mis en jeu au cours des cycles sexuels a permis de mettre au point des traitements hormonaux 
afin de faciliter la maîtrise de la reproduction (Grimard et al 2003). Le groupage des chaleurs qui en résulte permet une optimisation des conditions de travail. Utilisée en élevage, cette pratique repose sur la maîtrise de la durée de la phase lutéale et du contrôle du moment de la décharge pré-ovulatoire de LH. La synchronisation permet d'inséminer des groupes d'animaux le même jour, permettant de mieux surveiller d'éventuels retours 21 jours plus tard et de planifier les périodes de vêlage. Le contrôle du cycle sexuel s'appuie ainsi sur deux principes : le contrôle de la croissance folliculaire et celui de la durée de vie du corps jaune ou de la phase d'imprégnation de progestérone. Il existe trois types de traitements hormonaux, le plus utilisé étant celui à base de progestagènes ( $d$ 'après Grimard et al 2003).

\section{a) La PGF $2 \alpha$ ou analogues}

Le traitement consiste à effectuer deux injections de prostaglandines $\mathrm{PGF}_{2 \alpha}$ (ou analogues) à 11-14 jours d'intervalle, entre le 5ème et le 17 ème jour du cycle oestrien. Ces hormones provoquent la lutéolyse, mais n'ont pas d'effet direct sur la croissance folliculaire. La phase de chaleurs suivant la deuxième injection se déroulent ainsi sur plusieurs jours. L'insémination pendant ces chaleurs améliore les résultats de fertilité, mais ces traitements sont réservés aux animaux cyclés. D'après Folman et al (1990), le taux de gestation diminue lorsque le rang de lactation augmente. Les PGF $_{2 \alpha}$ seraient donc plus efficace sur génisses que sur multipares.

\section{b) L'association $\mathrm{GnRH} / \mathrm{PGF} \mathrm{F}_{2 \alpha}$}

La combinaison $\mathrm{GnRH} / \mathrm{PGF}_{2 \alpha}$ agit sur la croissance folliculaire et la lutéolyse et peut être utilisée sur des animaux cyclés ou non. Les chaleurs qui apparaissent après le traitement sont groupées, mais la fertilité à cet oestrus induit est variable (de 20 à 70\%). Des facteurs de variation (jour du cycle au moment du traitement, note d'état corporel...) sont connus mais difficilement maîtrisables au moment de l'application. Ces traitements ne sont pas conseillés pour les génisses. En effet, selon les résultats issus des essais menés par Pursley et al (1997), cette association a une efficacité réduite par rapport à un simple traitement de $\mathrm{PGF}_{2 \alpha}$, pour un coût plus élevé.

\section{c) Les progestagènes}

Ce dernier groupe de traitements libère des progestagènes associés à un œstrogène et/ou des $\mathrm{PGF}_{2 \alpha}$ et de 1'eCG (Equine Chorionic Gonadotropin). Les progestagènes sont utilisés pour induire l'oestrus chez les génisses prépubères. L'augmentation de progestérone qui survient avant l'initiation de la puberté chez la génisse est présumée être un prérequis au développement de cycles oestriens normaux. Les progestagènes stimulent la croissance du follicule et donc, l'augmentation de la production d'oestrogènes par les follicules ovariens (Patterson et al 1992). La durée de l'intervalle entre le retrait de l'implant et l'insémination est plus courte chez les génisses que chez les vaches. Une seule insémination est effectuée à $48 \mathrm{~h}$ au lieu d'une à $56 \mathrm{~h}$ ou de deux (une à $48 \mathrm{~h}$ et l'autre à $72 \mathrm{~h}$ ). Ces traitements peuvent être utilisés sur des animaux cyclés ou en anoestrus et présentent la même variabilité que les précédents. Cette méthode de synchronisation donne de bons résultats sur les génisses.

Pour plus de détails et de compréhension sur les intérêts et l'utilisation pratique de ces techniques, la lecture de l'article de Grimard et al (2003) est fortement recommandée.

\section{6 / Optimiser la croissance à moindre coût}

L'optimisation et la réduction des coûts d'élevage passent, comme pour la vache laitière, par une part de pâturage importante dans l'alimentation des animaux. Et même dans des systèmes relativement intensifs, Miller et Amos (1986) ont démontré qu'il était possible d'élever des génisses au pâturage et ce, de manière économe. Par ailleurs, l'utilisation d'herbe conservée (foin ou ensilage), voire de paille, avec peu de concentrés en période hivernale permet d'assurer une croissance satisfaisante pendant la période hivernale et de maîtriser le coût alimentaire, à condition de disposer par la suite de pâturages correctement utilisés.

\section{1 / Optimiser les possibilités de croissance compensatrice}

Les études menées aux USA par Ford et Park (2001) ont démontré que pour un même poids au vêlage, des vaches ayant eu une croissance linéaire depuis la naissance ont des performances laitières plus faibles que celles ayant présenté des croissances discontinues (figure 7). Selon ces auteurs, l'intérêt de cette pratique, basée sur les possibilités de croissance compensatrice, est évident et il est important d'identifier de manière plus fine quels sont les stades de compensation ou de repos. Ces essais ont été menés dans des conditions particulières (alimentation à base de maïs, animaux élevés en bâtiment, utilisation d'hormones de croissance recombinantes en lactation...), fréquemment rencontrées dans les conditions nord-américaines. Dans les conditions de production généralement rencontrées dans l'Ouest de l'Europe, ces situations sont rares. Une bonne adaptation des apports énergétiques, au bon moment et à la bonne quantité, serait ainsi bénéfique aux performances non seulement de croissance, mais aussi de production, de reproduction et de longévité lorsque la génisse devient adulte (Ford et Park 2001). Le coût d'élevage de la génisse serait alors optimisé, grâce aux possibilités de phase de moindre croissance permettant l'utilisation de fourrages et de concentrés de qualité moyenne en alternance avec des aliments plus coûteux. Sur le long terme, si cela permet une meilleure maîtrise du renouvellement et des réformes, c'est aussi une amélioration importante pour l'élevage tout entier qui peut être envisagé. Mais il faut noter que dans les travaux de Ford et Park (2001), seules les performances de production laitière ont été étudiées et que, vu le nombre limité d'animaux, il n'existe pas d'information sur les performances de reproduction, ni sur la carrière des vaches. Il convient donc d'approfondir ces approches et d'adapter ces pratiques aux conditions d'élevages européennes, pour tirer bénéfice de toutes ces capacités de croissance compensatrice chez la génisse vêlant à 24 mois.

Dans le cas du vêlage à 36 mois, des expériences menées à la station du Pin au Haras dans l'Orne ont permis d'étudier l'intérêt de différents niveaux de croissance au pâturage, induits après des plans d'alimentation maîtrisés et contrôlés pendant la période hivernale (Le Cozler et al 2009b). Au cours des années 89 à 93 , les plans d'alimentation utilisés ont permis des croissances de $800(\operatorname{lot} 1), 600(\operatorname{lot} 2)$ ou $400(\operatorname{lot} 3) \mathrm{g} / \mathrm{j}$ au cours des hivers 2 et 3 , pour des génisses nées à l'automne et au début de l'hiver 1. Ceci s'est traduit par des profils de croissance différents (figure 8), mais au final, un poids similaire après vêlage. Par comparaison au niveau haut (lot 1), des économies de 270 et $500 \mathrm{~kg}$ d'aliment concentrés ont été réalisées dans les lots 2 et 3 respectivement, ainsi que 80 et $150 \mathrm{~kg}$ de tourteau de soja. Les analyses effec- 
Figure 7. Intérêt de la modulation des plans de croissance de 6 à 34 mois sur les performances de lactation (d'après Ford et Park 2001).

\section{Poids vif, $\mathbf{k g}$}

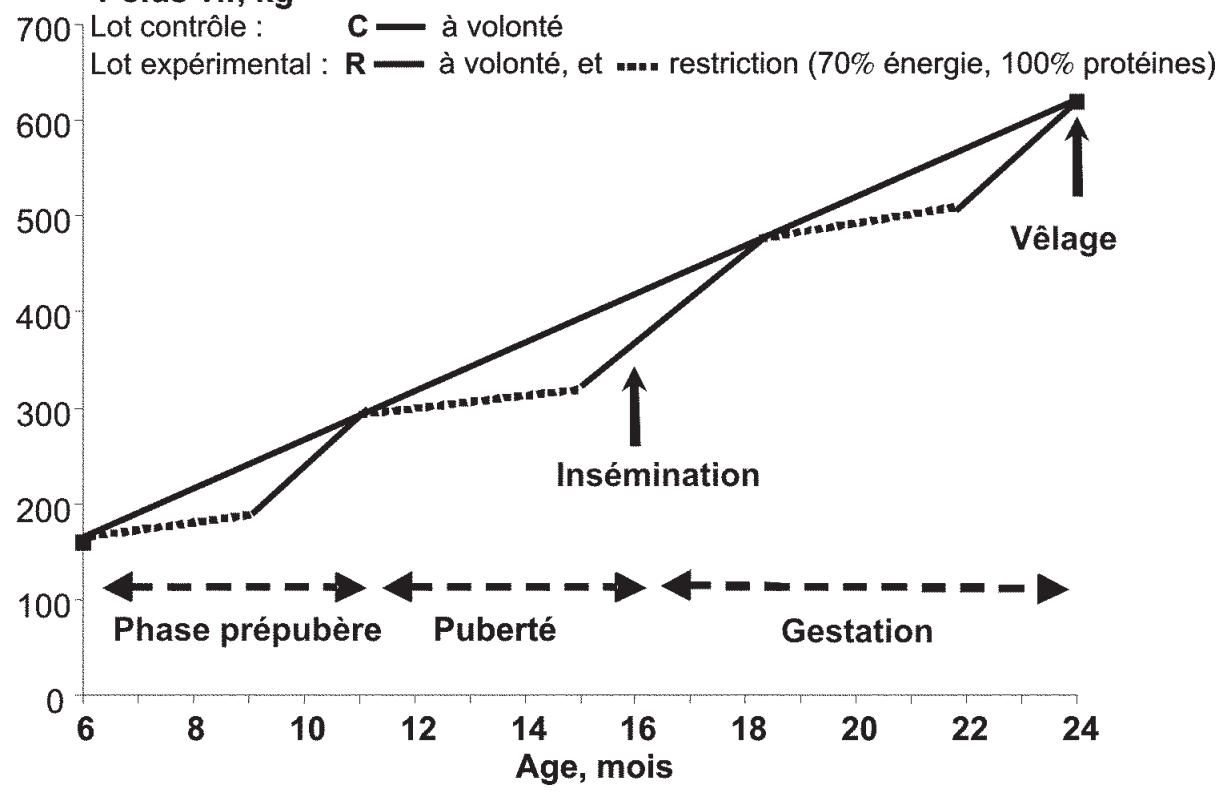

\begin{tabular}{|c|c|c|}
\hline & Lot expérimental R & Lot contrôle C \\
\hline Lactation 1, $\mathrm{n}$ & 4 & 4 \\
Kg de lait/j & $24,7^{\mathrm{a}}$ & $30,0^{\mathrm{b}}$ \\
\% protéines & 2,98 & 3,08 \\
\% lipides & 31,6 & 3,07 \\
\hline Lactation 2, & 3 & 3 \\
Kg de lait/j & $25,7^{\mathrm{a}}$ & $29,6^{\mathrm{b}}$ \\
\% protéines & 3,12 & 3,08 \\
\% lipides & 3,77 & 3,65 \\
\hline
\end{tabular}

Au sein d'une même ligne, deux chiffres avec une lettre différente diffèrent à $P<0,05$.

tuées ont montré qu'une croissance trop faible au cours de l'hiver tend à être pénalisante pour la fertilité des génisses $(75,69$ et $53 \%$ de réussite à l'IA1 pour les lots 1,2 et 3 respectivement). Mais les économies importantes dans le lot 3 ont été pénalisantes sur la durée de vie des animaux : moins d'un quart d'entre eux ont démarré une 3 ème lactation alors que plus de $30 \%$ des animaux des lots 2 et 3 ont produit au moins 4 lactations. Les niveaux de production ont également été affectés : en moyenne, $500 \mathrm{~kg}$ de lait en moins par lactation pour les animaux du lot 3 par rapport à ceux des niveaux 1 et 2 . Par la suite, des plans encore plus sévères $(600,400$ et $200 \mathrm{~g} / \mathrm{j})$ ont été mis en place mais seulement pendant l'hiver 2 à la Station du Pin au Haras, ainsi que des restrictions plus drastiques à Mirecourt (Vosges, 400, 200 et $0 \mathrm{~g} / \mathrm{j}$ ). Les analyses sur les possibles effets à long terme sont en cours d'analyse, et un bilan économique global sera réalisé prochainement.

Les précédentes analyses ont été réalisées dans des conditions proches de celles rencontrées en élevages, c'està-dire, en raisonnant par période (hiver $v s$ été), et non pas en fonction de l'âge de l'animal. Néanmoins, à l'issue de ces essais, une nouvelle série d'analyses effectuée sur une base individuelle devrait permettre de mieux mettre en évidence l'effet de la restriction en fonction de l'âge de l'animal et de tenir compte sans doute de la grande variabilité existante. Enfin, l'intérêt de croissance compensatrice également utilisée dans le cas du vêlage à 24 mois mériterait d'être vérifié avec ce type de dispositif expérimental.

\section{2 / Le pâturage chez la génisse (données actualisées à partir d'observations terrains)}

L'herbe pâturée assure des croissances compatibles avec un vêlage à 24 mois, avec par exemple des recommandations de $2 \mathrm{~kg} / \mathrm{MS} / 100 \mathrm{~kg} \mathrm{PV}$ (ingestion d'une génisse au printemps). Cette pratique nécessite de connaître la pousse de l'herbe. D'autres références font état de besoins d'un are par mois d'âge, valable uniquement pour une prairie fortement fertilisée (250 unités d'azote à l'hectare) produisant 9000 à $10000 \mathrm{~kg} \mathrm{MS} / \mathrm{ha}$. Cependant, dans le cadre d'un suivi réalisé sur une quinzaine d'élevages en Bretagne, Porhiel (2008b) n'observe plus cette valeur référence d'un are par mois d'âge, mais plutôt des valeurs de 1,5 à 2 . L'intérêt de ce suivi pluriannuel a été d'estimer le rendement des prairies utilisées par les génisses et ceci permet de mettre en relation ces valeurs avec les évolutions importantes des pratiques de fertilisation, avec des apports azotés nettement moins élevés que par le passé. En prenant en compte cette évolution, on s'aperçoit qu'au final, la référence de un are par mois d'âge, à condition de l'adapter en fonction des rendements des prairies et des régions, est toujours d'actualité. Ainsi, même avec une prairie de moindre qualité, il est possible de mettre en place un vêlage à 24 mois qui nécessite un niveau de croissance élevée, en adaptant le chargement et éventuellement, en mettant en place une complémentation adéquate. Ceci signifie bien qu'un suivi particulier des animaux est nécessaire si on veut qu'au final, les objectifs de croît soient atteints, quelles que soient les conditions d'alimentation.

La mise à l'herbe des jeunes animaux peut être précoce, notamment dans le cas de vêlages groupés au printemps. Des essais issus de la station de Trévarez dans le Finistère montrent ainsi que, sur 3 années, la sortie sur une prairie dès 15 jours d'âge des veaux allaités disposant de concentrés et de foin à volonté et d'un abri simple contre le vent et la pluie, se traduit par des performances comparables la première année et une croissance compensatrice plus élevée par la suite, comparativement aux veaux sortant à l'herbe à 6 mois d'âge (Porhiel 2008c). Dans un autre essai, Troccon et al (1997) notent des différences dans en termes de valorisation de l'herbe pâturée entre les races Normande et Holstein. Après la période de 0 à 14 mois où les croissances étaient modérées (664 à $702 \mathrm{~g} / \mathrm{j}$, groupe $\mathrm{M})$ ou importantes (819 à $862 \mathrm{~g} / \mathrm{j}$, groupe $\mathrm{H}$ ), les génisses Normande du groupe $\mathrm{M}$ ont montré une plus grande capacité à mieux utiliser l'herbe que les génisses Holstein de ce même groupe, ce qui s'est traduit par de meilleures croissances. Au final, aucune différence de poids vif au $1^{\text {er }}$ vêlage n'est observée entre vaches Normande des lots $\mathrm{M}$ et $\mathrm{H}$. Au contraire, il existe une différence de $+26 \mathrm{~kg}$ $(\mathrm{P}<0,05)$ en faveur des vaches Holstein du groupe $\mathrm{H}$ par rapport à 
Figure 8. Influence de restrictions alimentaires pendant les phases hivernales sur les profils de croissance et les performances de génisses laitières (Normande et Holstein) vêlant à 36 mois (Le Cozler et al 2009b).

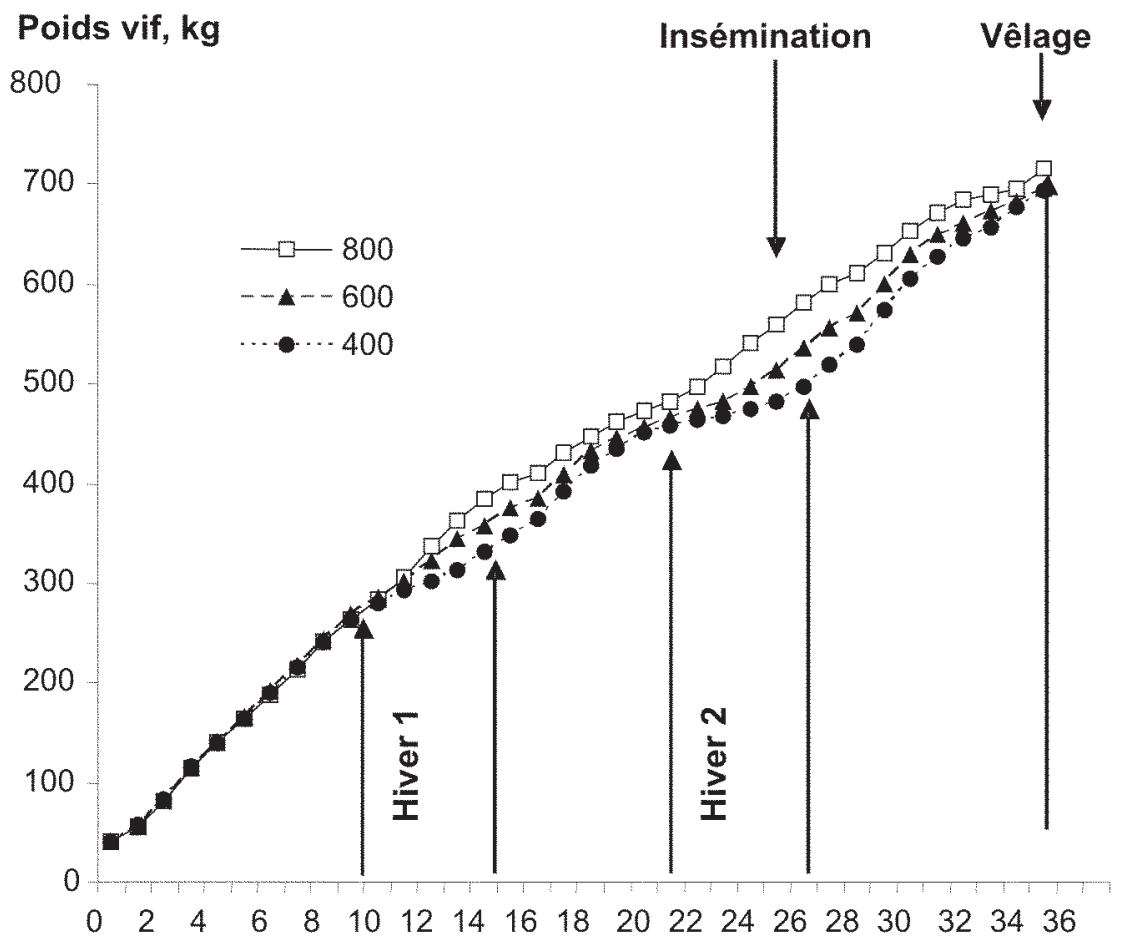

Age, mois

\begin{tabular}{|l|c|c|c|}
\hline $\begin{array}{l}\text { Objectifs de croissance pendant } \\
\text { l'hiver, g/j }\end{array}$ & $\mathbf{8 0 0}$ & $\mathbf{6 0 0}$ & $\mathbf{4 0 0}$ \\
\hline Effectif & 70 & 69 & 70 \\
\hline $\begin{array}{l}\text { Production de lait (base 305 j), kg } \\
\text { Lactation 1 }\end{array}$ & $\begin{array}{c}6178 \\
7550^{\mathrm{a}}\end{array}$ & $\begin{array}{c}5980 \\
7303^{\mathrm{b}}\end{array}$ & $\begin{array}{c}6152 \\
\text { Lactation 2 et plus }\end{array}$ \\
\hline Nombre de lactations avant réforme & $2,2^{\mathrm{a}}$ & $1,9^{\mathrm{ab}}$ & $1,5^{\mathrm{b}}$ \\
\hline
\end{tabular}

Au sein d'une même ligne, deux chiffres avec une lettre différente diffèrent à $P<0,05$

leurs homologues du groupe M. Cette différence observée dans les années 70 a été confirmée 20 ans plus tard par Charton (2008), sur les données issues de la station du Pin au Haras. Néanmoins, l'existence d'une telle différence entre ces deux races n'a pu être démontrée chez les animaux adultes.

\section{Conclusion}

La maîtrise de l'âge au premier vêlage (24, 30 ou 36 mois) selon le système de fourrage est cruciale. Des pratiques cohérentes avec l'objectif d'âge au $1^{\mathrm{er}}$ vêlage retenu sont essentielles. Dans le cas d'un vêlage à 24 mois d'âge, une puberté acquise précocement, une cyclicité maintenue, un bon état d'engraissement et un bon développement aux différents stades sont importants. Ceux-ci dépendent grandement des programmes alimentaires et des races. Il existe néanmoins une grande variabilité de performances, de maturité... non seulement entre races et individus, mais aussi entre systèmes d'élevage. La prise en compte de cette variabilité est essentielle dans la gestion à moyen et court terme des politiques de mise à la reproduction des génisses.

Les effets de l'utilisation de la croissance compensatrice pratiquée en vue de maîtriser le coût alimentaire, sur la fertilité et la longévité des vaches constituent une piste intéressante de travail, mais ils doivent être testés en termes de faisabilité en élevage. Dans le cas d'un vêlage à 30 ou 36 mois des rythmes de croissance plus modérés en période de stabulation sont possibles à condition de ne pas descendre trop bas en terme de croissance journalière $(400 \mathrm{~g} / \mathrm{j}$, à confirmer), pour ne pas affecter la reproduction et la carrière des vaches, et de disposer de pâtures bien exploitées pour bénéficier de croissance comvêlages groupés ou pour la conduite en lots, la pratique alimentaire peut être pensatrice par la suite. Dans le cas de accompagnée de traitements hormonaux ( $\mathrm{PGF}_{2 \alpha}$ ou analogues, progestagènes).

Des travaux de recherche sont engagés pour étudier la faisabilité d'un vêlage encore plus précoce que le vêlage à 24 mois, de l'ordre de 20 à 21 mois, dans des systèmes laitiers français à terres labourables, qui permettrait de réduire notablement l'effectif de génisses à élever. Cette pratique, entraînant une puberté très précoce, nécessite une croissance élevée mais non engraissante, avec sans doute des niveaux d'apports en protéines à adapter.

On peut résumer ainsi l'élevage de la génisse en fonction de différents objectifs, avec les deux extrêmes d'âge au vêlage (les autres ayant des situations intermédiaires à adapter au cas par cas) :

1) Pour un élevage précoce (par exemple 24 mois, en race Holstein), il faut viser :

- $200 \mathrm{~kg}$ à 6 mois : la croissance élevée $(900 \mathrm{~g} / \mathrm{j})$ a un effet favorable sur le développement de la génisse et la longévité de la vache laitière ;

- $400 \mathrm{~kg}$ à 15 mois : avec un GMQ de 1 'ordre $750 \mathrm{~g} / \mathrm{j}$, de 6 à 15 mois en jouant aussi sur les capacités de croissance compensatrice ;

- $600 \mathrm{~kg}$ au $1^{\mathrm{er}}$ vêlage : avec un GMQ de $750 \mathrm{~g} / \mathrm{j}$, cela permet d'éviter les problèmes de vêlage et d'optimiser la production en 1ère lactation.

2) Pour un élevage tardif (par exemple 36 mois, en race Normande), il faut faire attention à :

- obtenir une croissance élevée $(900 \mathrm{~g} / \mathrm{j})$ de la naissance à 4-6 mois d'âge, comme précédemment. Ceci a un effet favorable sur le développement de la génisse et la longévité de la vache laitière ;

- à avoir une croissance modérée ensuite $(500 \mathrm{~g} / \mathrm{j})$, jusqu'au dernier tiers de gestation, afin de ne pas risquer un état d'engraissement trop pénalisant ;

- et penser que l'on peut moduler la fin de gestation (croissance $>500 \mathrm{~g} / \mathrm{j}$ ) afin de compenser les effets trop néfastes d'éventuelles carences importantes en cours de croissance.

Dans le premier cas et pour des races précoces sexuellement, le poids à la puberté sera de l'ordre de 40 à $50 \%$ de poids adulte, contre 50 à $55 \%$ dans le second. De même, les différences de potentiel de croissance, de maturité... se traduiront par un poids au $11^{\mathrm{er}}$ vêlage voisin respectivement de $90-95 \%$ et 85 $90 \%$ du poids adulte. 


\section{Références}

Agabriel J., Meschy R.F., 2007. Alimentation des veaux et génisses d'élevage. In : Alimentation des bovins, ovins et caprins. Besoins des animaux. Valeurs des aliments, Tables INRA 2007. Editions Quae, Versailles, France, 75-87.

Augeard P., Bazin S., Disenhaus C., 1986. Pathologie post-partum dans des troupeaux à forte production de l'Ouest de la France. I. Hiérarchie et associations pathologiques. Interactions entre pathologie, reproduction et production. Proc. XIVth World Congr. Diseases of Cattle, Dublin, Irlande, 1386-1391.

Barbat A., Bonatti F., Guillaume F., Druet T., Colleau J.J., Boichard D., 2005. Bilan phénotypique de la fertilité à l'insémination artificielle dans les trois principales races laitières françaises. Renc. Rech. Rum., 12, 137-140.

Barbat A., Gion A., Ducrocq V., 2007. L'évaluation génétique de la fertilité en France, B.T.I.A., 126, 19-22.

Bar-Peled U., Robinzon B., Maltz E., Tagari H., Folman Y., Bruckental I., Voet H., Gacitua H., Lehrer A.R., 1997. Increased weight gain and effects on production parameters of Holstein heifer calves that were allowed to suckle from birth to six weeks of age. J. Dairy Sci., 80, 2523-2528.

Brisson J., 2006. Le remplacement stratégique des vaches dans le troupeau. 30 ème Symp. sur les bovins laitiers, CRAAQ, St-Hyacinthe, Québec, Canada., décembre, 19p.

Brunschwig P., Porhiel J.Y., Jégou V., Bertin M., Plouzin D., Blois M., 2005. Référentiel élevage du veau. Groupe Interrégional génisses laitières, Institut de l'Elevage, Chambres d'Agriculture, Contrôle Laitier, Bovins Croissance des régions Bretagne, Pays de la Loire et Basse-Normandie, 51p.

Byerley D.J., Staigmiller R.B., Berardinelli J.G., Short R.E., 1987. Pregnancy rates of beef heifers bred either on pubertal or third oestrus. J. Anim. Sci., 65, 645-650.

Chagas L.M., Rhodes F.M., Blache D., Gore P.J.S., MacDonald K.A., Verkerk G.A., 2006. Precalving effects on metabolic responses and postpartum anoestrus in grazing primiparous dairy cows. J. Dairy Sci., 89, 1981-1989.

Charton C., 2008. Croissance et fertilité chez la génisse laitière. Rapport de fin d'études, IUT d'Agronomie de Brest, France, 45p.

Chavatte-Palmer P., Al Gubory K., Picone O, Heyman Y., 2008. Nutrition maternelle : incidence sur la fertilité de la descendance et importance de la période périconceptionelle pour le long terme. Gynécol. Obst. Fertil., 36, 920-929.

Corpen, 1999. Estimation des flux d'azote, de phosphore et de potassium associés aux vaches laitières et à leur système fourrager : influence de l'alimentation et du niveau de production. Ministère de l'agriculture et de la pêche; Ministère de l'aménagement du territoire et de l'environnement, CORPEN, Comité d'orientation pour la réduction de la pollution des eaux par les nitrates, les phosphates et les produits phytosanitaires provenant des activités agricoles, Groupe Alimentation animale, Sous groupe Vaches laitières. Paris, France, 18p.

Dardillat J., Trillat G., Larvor P., 1978. Colostrum immunoglobulin concentration in cows: relationship with their calf mortality and with the colostrum quality of their female offspring. Ann. Rech. Vét., 9, 375-384.
D'Hour P, Coulon J.B, Petit M, Garel J.P. 1995. Caractérisation zootechnique de génisses de races Holstein, Montbéliarde et Tarentaise. Ann. Zootech., 44, 217-227.

Disenhaus C., 1991. Génisses : croissance et carrière. Bull. GTV, 4B, 383, 25-37.

Disenhaus C., Bazin S., Augeard P., 1986. Pathologie post-partum dans des troupeaux à forte production de l'Ouest de la France. II. Effets des états d'engraissement et de leurs évolutions sur la pathologie post-partum. Proc. $X^{\text {XIth }}$ World Congr. Diseases of Cattle, Dublin, Irlande, 1380-1385.

Disenhaus C., Grimard B., Trou G., Delaby L., 2005. De la vache au système : s'adapter aux différents objectifs de reproduction en élevage laitier ? Revue de synthèse. Renc. Rech. Rum., $12,125-136$

Dozias D., Peccatte J.R., Michel G., 2006. Influence du profil de croissance sur les performances de reproduction de génisses charolaises de 2 ans d'âge. Renc. Rech. Rum., 13, 287.

Erb H.N., Smith R.D., Oltenacu P.A., Guard C.L., Hillman R.B., Powers P.A., Smith M.C., White M.E., 1985. Path model of reproductive disorders and performance, milk fever, mastitis, milk yield and culling in Holstein cows. J. Dairy Sci., 68, 3337-3349.

Foley J.A., Otterby E., 1978. Availability, storage, treatment, composition and feeding value of surplus colostrum: a review. J. Dairy Sci., 6, 1033-1060.

Folman Y., Kaim M., Herz Z., Rosenberg M., 1990. Comparison of methods for the synchronization of estrous cycles in dairy cows. 2. Effects of progesterone and parity on conception. J. Dairy Sci., 73, 2817

Ford J.A., Park C.S., 2001. Nutritionally directed compensatory growth enhances heifer development and lactation potential. J. Dairy Sci., 84, 1669-1678.

Frisch R.E., 1984. Body fat, puberty and fertility. Biol. Rev., 59, 161-168.

Gardner D.S., Lea R.G., Sinclair K.D., 2008. Developmental programming of reproduction and fertility: what is the evidence? Animal, 2, 1128-1134.

Gasser C.L., Behlke E.J., Grum D.E., Day M.L., 2006. Effect of timing of feeding a highconcentrate diet on growth and attainment of puberty in early-weaned heifers. J. Anim. Sci., $84,3118-3122$.

Gauthier D., Nerot F., Garel J.P., Petit M. 1986. Etude de la puberté chez la génisse Salers. Influence de certains paramètres de l'environnement. Bull. Tech. CRZV Theix, INRA, $64,55-58$.

Grimard B., Disenhaus C., 2005. Les anomalies de reprise de cyclicité après vêlage. Le Point Vétérinaire, 36, 16-21

Grimard B., Humblot P., Ponter A.A., Chastant S., Constant F., Mialot J.P., 2003. Efficacité des traitements de synchronisation des chaleurs chez les bovins. INRA Prod. Anim., 16, 211-227.

Gulliksen S.M., Lie K., Solverod L., Osteras O., 2008. Risk factors associated with colostrum quality in norwegian dairy cows. J. Dairy Sci., 91, 704-712.
Hadley G.L., Wolf C.A., Harsh S.B., 2006. Dairy cattle culling patterns, explanations, and implications. J. Dairy Sci., 89, 2286-2296.

Heinrichs A.J., 1996. Nutrition and management of replacement cattle. Anim. Feed Sci. Technol., 59, 155-166.

Hoch T., Begon C., Cassar-Malek I., Picard B., Svary-Auzeloux I., 2003. Mécanismes et conséquences de la croissance compensatrice chez les ruminants. INRA Prod. Anim., 16, 49-

Hornick J.L., Van Eenaeme C., Gérard O Dufrasne I., Istasse L., 2000. Mechanisms of reduced and compensatory growth. Dom. Anim. Endocrinol., 19, 121-132.

Hohenboken W.D., Foldager J., Jensen J., Madsen P., Andersen B.B., 1995. Breed and nutritional effects and interactions on energy intake, production and efficiency of nutrient utilization in young bulls, heifers and lactating cows. Acta Agric. Scand., Section A, Anim. Sci., $45,92-98$.

Huxley J.S, 1932. Problems of relative growth. Methuen and Co (Ed), London, UK $276 \mathrm{p}$.

INRA, 2007. Alimentation des bovins, ovins et caprins. Besoins des animaux. Valeurs des aliments, Tables INRA 2007. Editions Quae, Paris, France, $307 \mathrm{p}$.

Kaur H., Arora S.P., 1995. Dietary effects on ruminant livestock reproduction with particular reference to protein. Nutr. Res. Rev., 8, 121-136.

Lacau-Mendigo I.M., Mejia M.E., Diaz-Torga G.S., Gonzalez Iglesias A., Formia N., Libertun C., Becu-Villalobos D., 2000. Endocrine studies in ivermectin-treated heifers from birth to puberty. J. Anim. Sci., 78, 817-824.

Le Cozler Y., Le Dividich J., 2004. L'hyperprolificité: conséquences sur la truie et les porcelets. Colloque sur la production porcine, 25 ans d'évolution, CRAAQ, St-Hyacinthe, Québec, Canada, 91-123.

Le Cozler Y., Ringmar-Cederberg E., Johansen S., Dourmad J.Y., Neil M., Stern S., 1999. Effect of feeding level during rearing and mating strategy on performance of Swedish Yorkshire sows. 1. Growth, puberty and conception rate. Anim. Sci., 68, 355-363.

Le Cozler Y., Lollivier V., Lacasse P. Disenhaus C., 2008. Rearing strategy and optimizing first calving targets in dairy heifers: a review. Animal, 2, 1393-1404.

Le Cozler Y., Peyraud J.L., Troccon J.L., 2009a. Effect of feeding regime, growth intensity and age at first insemination on performances and longevity of Holstein heifers born during autumn. Livest. Sci., 124, 72-81.

Le Cozler Y., Peccatte J.R., Delaby L., 2009b. A comparative study of three growth profiles during rearing in dairy heifers: effect of feeding intensity during two successive winters on performances and longevity. Livest. Sci., sous presse.

Le Mat J.L., Bazire A., Mesnil C., Messager P., Troccon J.L., 1994. L'état d'engraissemen des génisses Prim'Holstein. Document EDE, Chambre d'agriculture de Bretagne, INRA, GIE Lait Viande, Bovins Croissance Bretagne, $4 \mathrm{p}$.

Lyford S.J., 1988. Growth and development of the ruminant digestive system. In: The ruminant 
animal digestive physiology and nutrition. D.C Church (Ed), Practice Hall, Englewood Cliffs, New Jersey, USA, 44.

Lopez-Gatius F., Yaniz J., Madriles-Helm D., 2003. Effects of body condition score and score change on the reproductive performance of dairy cows: a meta-analysis. Theriogenology, 59, 801812.

Martin J.L., Vonnahme K.A., Adams D.C., Lardy G.P., Funston R.N., 2007. Effects of dam nutrition on growth and reproductive performance of heifer calves. J. Anim. Sci., 85, 841-847.

Miller W.J., Amos H.E., 1986. Feeding dairy heifers in the current economic climate. Feedstuffs, 10, 28.

Mourits M.C.M., Huirne R.B.M., Dijkhuizen A.A., Kristensen A.R., Galligan D.T., 1999. Economic optimization of dairy heifer management decisions. Agric. Syst., 61, 17-31.

Muller L.D., Ellinger D.K., 1981. Colostral immunoglobulin concentrations among breeds of dairy cattle. J. Dairy Sci., 64, 1727-1730.

Patterson D.J., Perry R.C., Kiracofe G.H., Bellows R.A., Stagmiller R.B., Corah L.R., 1992. Management considerations in heifer development and puberty. J. Anim. Sci., 70, 4018-4035.

Pavaux X., 1981. Eléments d'anatomie. In L'utérus de la vache, anatomie, physiologie, pathologie, Constantin et Meissonnier (Eds), Société francaise de Buiatrie, ENV d'Alfort, Maisons Alfort, France, 9-52.

Peccate J.R., Michel G., Delaby L., Troccon J.L., 2006. Influence du profil de croissance autour de la période d'insémination sur la fertilité des génisses laitières de race Holstein et Normande conduites en vêlage 3 ans. Renc. Rech. Rum., 13, 286.

Peri I., Gertler A., Bruckental I., Barash A., 1993. The effect of manipulation in energy allowance during the rearing period of heifers on hormone concentrations and milk production in first lactation cows. J. Dairy Sci., 76, 742-751.

Philipot J.M. 2008. Les conditions d'élevage et la baisse de fertilité. BTIA, 128, 51-52.

Philipson J., 1976. Studies in calving difficulty, stillbirth and associated factors in Swedish cattle breeds. IV. Relationship between calving performances, precalving body measurements and size of pelvic opening in Friesian heifers. Acta Agric. Scand. Section A, Anim. Sci., 26, 221-226.

Pierre S., 2003. Exploration de nouveaux repères pour le pilotage de l'élevage des génisses laitières. Rapport de fin d'étude DESS, Université Rennes1 / Ensar.

Pirlo G., Capelleti M., Marchetto G., 1997. Effects of energy and protein allowances in the diets of prepubertal heifers on growth and milk production. J. Dairy Sci., 80, 730-739.

Porhiel J.Y., 2002. Travail et génisses laitières : le temps passé dépend avant tout de l'éleveur. A la pointe de l'élevage, 402, 8-10.

Porhiel J.Y., 2008a. Elevage des veaux : l'utilisation de ferments lactiques simplifie l'élevage. Cap Elevage, 30, 22.

Porhiel J.Y., 2008b. Vêlage précoce des génisses laitières: recommandations alimentaires au pâturage. Cap Elevage, 29, 26-29.

Porhiel J.Y., 2008c. Du travail en moins : le pâturage précoce des petits veaux. Cap Elevage, $22,10-11$

Pritchett L.C., Gay C.C., Besser T.E., Hancock D.D., 1991. Management and production factors influencing immunoglobulin G1 concentration in colostrum from Holstein cows. J. Dairy Sci., 74, 2336-2341.

Pursley J.R., Wiltbank M.C., Stevenson J.S., Ottobre J.S., Garverick H.A., Anderson L.L., 1997. Pregnancy rates per artificial insemination for cows and heifers inseminated at a synchronized ovulation or synchronized estrus. J. Dairy Sci., 80, 295-300.

Reid J.T., Loosli J.K., Trimberger G.W., Turk K.L., Asdell S.A., Smith S.E., 1964. Causes and prevention of reproductive failures in dairy cattle. IV. Effect of plane of nutrition during early life on growth, reproduction, health and longevity of Holstein cows. 1. Birth to fifth calving. Cornell University Agricultural Experiment Station Bulletin 987. Ithaca, New York, USA, 31p.

Rémond B., Kérouanton J., Brocard V., 1997. Effets de la réduction de la période sèche ou de son omission sur les performances des vaches laitières. INRA Prod. Anim., 10, 301-315.

Robelin J., 1986. Bases physiologiques de la production de viande : croissance et développement des bovins. In: Production de viande bovine. D. Micol (Ed), INRA publications, Paris, France, 35-60.

Swali A., Wathes D.C., 2007. Influence of primiparity on size at birth, growth, somatotrophic axis and fertility in dairy heifers. Anim. Reprod. Sci., 102, 122-136.

Troccon J.L., 1984. Lait entier et colostrum pour les veaux d'élevage. Bull. Tech. CRZV, Theix, 57, 57-60.

Troccon J.L., 1989. Allaitement et sevrage des génisses d'élevage. INRA Prod. Anim., 2, 189. 195.

Troccon J.L., 1996. Elevage des génisses laitières et performances ultérieures. Renc. Rech. Rum., 3, 201-210.

Troccon J.L., Petit M., 1989. Croissance des génisses de renouvellement et performances ultérieures. INRA Prod. Anim., 2, 55-64.

Troccon J.L., Toullec R., 1989. Aliments d'allaitement pour veaux d'élevage. INRA Prod. Anim., 2, 117-128.

Troccon J.L., Muller A., Peccatte J.R., Fargetton M., 1997. Effet du niveau d'alimentation énergétique de génisses laitières de races Holstein et Normande jusqu'à l'âge de 14 mois sur les performances durant les périodes d'élevage et de lactation. Ann. Zootech., 46, 27-41.

Walsh R.B., Kelton D.F., Duffield T.F., Leslie K.E., Walton J.S., Leblanc S.J., 2007. Prevalence and risk factors for postpartum anovulatory condition in dairy cows. J. Dairy Sci., 90, 315-324.

Wattiaux M., 1997. L'essentiel Laitier, Institut Babcock, Wisconsin University, USA, 140p.

Weaver D.M., Tyler J.W., VanMetre D.C., Hostetler D.E., G.M. Barrington, 2000. Passive transfer of colostral immunoglobulins in calves. J. Vet. Internal Med., 14, 569-577.

\section{Résumé}

En élevage laitier, l'éleveur doit réussir à amener ses génisses de renouvellement à être de bonnes femelles laitières (notamment des points de vue de la production, de la reproduction et de la longévité), adaptées aux conditions d'élevage et au système de production, et ce, au moindre coût de production. Ceci reste une gageure, mais des solutions existent. Le présent article se propose de faire le point sur cellesci, en s'intéressant particulièrement aux conditions d'élevage et aux différentes étapes clés de l'élevage de la génisse laitière, de sa naissance jusqu'au premier vêlage. Certaines informations allant au-delà (réforme par exemple) complètent cette approche. L'article reprend et réactualise les données existantes depuis plusieurs décennies, notamment en ce qui concerne l'alimentation de la future vache laitière. La maîtrise de l'âge au $1^{\mathrm{er}}$ vêlage $(24,30$ ou 36 mois dans le cas des vêlages groupés), selon le système de fourrage considéré ou en place dans les élevages, s'avère cruciale. Dans le cas d'un vêlage à 24 mois d'âge, une puberté acquise précocement, une cyclicité maintenue, un bon état d'engraissement et un bon développement aux différents stades sont des objectifs à atteindre dans les meilleures conditions possibles pour le succès de cette pratique. Ceux-ci dépendent grandement des programmes alimentaires et des races, mais il est important d'éviter un engraissement excessif qui pourrait être pénalisant pour la suite de la carrière. Dans le cas d'un vêlage à 30 ou 36 mois des rythmes de croissance plus modérés, notamment en période de stabulation, sont possibles, à condition de ne pas descendre trop bas en terme de croissance journalière ( $400 \mathrm{~g} / \mathrm{j}$ ou moins), pour ne pas affecter la reproduction et la carrière des vaches. Les régimes alimentaires à base d'herbe et exploitant les capacités de croissance compensatrice des animaux permettent de réduire significativement et efficacement les coûts d'élevage. Dans le cas de vêlages groupés ou lors de la conduite en lots, la pratique alimentaire peut être accompagnée de traitements hormonaux ( $\mathrm{PGF}_{2 \alpha}$ ou analogues, progestagènes). Réduire encore plus l'âge au vêlage vers 20 mois d'âge, avec une réduction des coûts, des rejets, du travail... est sans doute possible et cette possibilité nécessitera d'être testée dans les années à venir. 


\begin{abstract}
Rearing dairy heifers

On dairy farms, rearing heifers is aimed at having efficient milking cows, from reproductive, productive and longevity points of views. The animals also need to be adapted to their living environment and to be reared at the lowest cost possible. Practical solutions exist and the present paper is an update of rearing dairy heifer procedures, from birth to first calving, and their subsequent performances. Age at first calving (24, 30 or 36 months of age in seasonal calving systems for example) is of importance when considering the dairy system. With first calving at $\mathbf{2 4}$ months of age, puberty should arise early and body development and reserve should be adequate. However, a too high growth rate should be avoided because of possible excessive fatness that could have a deleterious effect on long-term performances. With calving at 30 or 36 months of age, growth intensity could be reduced, particularly when animals are housed during the winter. But insufficient growth performances $(400 \mathrm{~g} / \mathrm{d}$ or less) could be deleterious for reproduction and longevity. The grazing system, optimising compensatory growth capacity of heifers, can reduce rearing costs significantly. When seasonal calving procedures are used in the herd, hormonal treatment could be useful. Reducing even more the age at first calving (20 months or less), resulting in low rearing and labour costs... is probably of interest but has to be studied in detail.
\end{abstract}

LE COZLER Y., PECCATTE J.-R., PORHIEL J.-Y., BRUNSCHWIG P., DISENHAUS C., 2009. Pratiques d'élevages et performances des génisses laitières : état des connaissances et perspectives. Inra Prod. Anim., 22, 303-316. 